\title{
Application of Smoluchowski's generalized theory to the kinetics of triplet-triplet annihilation of anthracene in viscous solution after long-pulse excitation
}

\author{
(the late)B. Nickel, $\dagger^{a}$ P. Borowicz, $f^{a}$ A. A. Ruth ${ }^{* b}$ and J. Troe ${ }^{a}$ \\ a Max-Planck Institut für Biophysikalische Chemie, Abteilung Spektroskopie und \\ Photochemische Kinetik, Am Fassberg 11, D-37077, Göttingen, Germany \\ ${ }^{b}$ Department of Physics, National University of Ireland, University College Cork, Cork, \\ Ireland.E-mail: a.ruth@ucc.ie; Tel: +353-21-4902057
}

Received 22nd December 2003, Accepted 31st March 2004

First published as an Advance Article on the web 24th May 2004

The validity of Smoluchowski's theory is demonstrated by its application to the bimolecular triplet-triplet annihilation (TTA) $T_{1}+T_{1} \rightarrow S_{0}+S_{1}$ in viscous solution. For that purpose the delayed $S_{1} \rightarrow S_{0}$ fluorescence of anthracene in cis-1,3-dimethylcyclohexane/trans-1,4-dimethylcyclohexane (1:1) was measured after UV excitation with long laser pulses $(\sim 30 \mu \mathrm{s})$ in a temperature range where TTA is completely diffusion controlled. Through the adequate treatment of the kinetics of TTA upon spatially homogeneous and spatially periodic excitation (which is partially based on the results of previous publications) absolute values for the diffusion coefficients $D$ of triplet molecules, for the effective annihilation distance $R_{\mathrm{A}}$ and for the Förster radius $R_{\mathrm{ST}}$ of heterogeneous annihilation $\mathrm{S}_{1}+\mathrm{T}_{1} \rightarrow \mathrm{S}_{0}+\mathrm{T}_{n}$ were determined. The temperature dependence of all three parameters $\left(D, R_{\mathrm{A}}\right.$ and $\left.R_{\mathrm{ST}}\right)$ is discussed. From the decrease of $R_{\mathrm{A}}$ with increasing temperature, the parameters $A$ and $L$ can be determined, which define the exponential distance dependence of the annihilation of a triplet pair in the kinetic model of Butler and Pilling (1977). Differences of the two models and applicability criteria are discussed.

\section{Introduction}

The first excited triplet state $\left(T_{1}\right)$ in aromatic compounds such as anthracene is generally populated by optical excitation of ground state $\left(\mathrm{S}_{0}\right)$ molecules to the first excited singlet state $\left(\mathrm{S}_{1}\right)$, from where subsequent radiationless intersystem crossing leads to the population of the lowest triplet state: $\mathrm{S}_{0} \rightarrow \mathrm{S}_{1} \leadsto \mathrm{T}_{1}$. In liquid solution metastable triplet states can decay in two different ways, provided quenching mechanisms (e.g. by molecular oxygen) are negligible:

(1) By first-order processes, i.e. phosphorescence and nonradiative decay, with a rate constant $k_{\mathrm{T}}$ :

$$
\mathrm{T}_{1} \underset{k_{\mathrm{T}}}{\stackrel{{ }^{2}}{\longrightarrow}} \mathrm{S}_{0} .
$$

(2) By diffusion-controlled triplet-triplet annihilation (TTA) with a second-order rate coefficient $k_{2 \mathrm{~A}}: \S$

$$
\begin{aligned}
\mathrm{T}_{1}+\mathrm{T}_{1} \stackrel{k_{2 \mathrm{~A}}}{\longrightarrow} \mathrm{S}_{0}+\mathrm{S}_{1} \\
\downarrow \\
\downarrow \\
\mathrm{S}_{0}+\nu_{\mathrm{DF}} .
\end{aligned}
$$

With a certain probability the annihilation of a triplet pair yields an excited singlet state $S_{1}$ and hence gives rise to a delayed fluorescence (DF) $S_{1} \rightarrow S_{0}$. From the time dependence of the DF all relevant information on diffusion-controlled TTA can be extracted, which is the main subject of this paper.

$\dagger$ Passed away on the 27/01/2002.

$\ddagger$ Present address: Institute of Physical Chemistry, Polish Academy of Sciences, Kasprzaka 44/52, 01-224 Warsaw, Poland.

$\S$ In this paper we adhere to the terminology and symbols used in a preceding paper, ${ }^{3}$ thus kinetic quantities referring to TTA are labelled with a subscript $\mathrm{A}$.
The rate coefficient $k_{2 \mathrm{~A}}$ is a time-dependent quantity according to Smoluchowski's theory ${ }^{1,2}$ of diffusion-controlled bimolecular reactions in solution. The time dependence of $k_{2 \mathrm{~A}}$ for TTA was the topic of a preceding paper, ${ }^{3}$ in which we showed that Smoluchowski's original theory can be applied to the kinetics of diffusion-controlled TTA, if the effect of the Förster energy transfer reactions (FRETs),

$$
\begin{gathered}
\mathrm{S}_{1}+\mathrm{S}_{1} \stackrel{k_{\mathrm{SS}}(r)}{\longrightarrow} \mathrm{S}_{0}+\mathrm{S}_{n}, \\
\mathrm{~S}_{1}+\mathrm{T}_{1} \stackrel{k_{\mathrm{ST}}(r)}{\longrightarrow} \mathrm{S}_{0}+\mathrm{T}_{m},
\end{gathered}
$$

is taken into account (see Fig. 2 in ref. 3). Since the reactions (3a) and (3b) strongly affect the primary formation of pairs of triplet molecules, $\mathrm{T}_{1} \cdots \mathrm{T}_{1}$, at short intermolecular distances $r$, $\uparrow$ Smoluchowski's approach must be modified in two ways for diffusion-controlled TTA in viscous solution: ${ }^{3}$

Modification 1: Originally Smoluchowski solved Fick's second law for a spherical problem,

$$
\frac{\partial \rho(r, t)}{\partial t}=\frac{D}{r} \frac{\partial^{2}[r \rho(r, t)]}{\partial r^{2}},
$$

with the boundary condition

$$
\rho(r, t)=0 \quad(t>0),
$$

where $\rho(r, t)$ is the time-dependent relative concentration of reactive particles in the neighbourhood of a selected reactive

9 The rate coefficients $k_{\mathrm{SS}}(r) \propto k_{\mathrm{S}}\left(R_{\mathrm{SS}} / r\right)^{6}$ and $k_{\mathrm{ST}}(r) \propto k_{\mathrm{S}}\left(R_{\mathrm{ST}} / r\right)^{6}$ are distance-dependent, where $k_{\mathrm{S}}$ is the first-order rate constant for the decay of $\mathrm{S}_{1}$ in the absence of an energy acceptor and $R_{\mathrm{SS}}$ and $R_{\mathrm{ST}}$ are the respective Förster radii. ${ }^{4,5}$ 
particle, $D$ is the relative diffusion coefficient of two reactive particles, and $R$ is the encounter distance of two reactive particles. ${ }^{1}$ For diffusion-controlled TTA the encounter distance $R$ in the boundary condition must be simply identified by an effective intermolecular annihilation distance $R_{\mathrm{A}}{ }^{3}$

Modification 2: Smoluchowski's initial condition, which defines a completely statistical spatial distribution of reactive particles at time $t=0$,

$$
\begin{array}{ll}
\rho(r, 0)=0 & (r \leq R), \\
\rho(r, 0)=1 & (r \geq R),
\end{array}
$$

has to be replaced by a new initial condition

$$
\rho_{\mathrm{A}}(r, 0)=\frac{p r^{6}}{\left(r^{6}+R_{\mathrm{ST}}^{6}\right)}+\frac{(1-p) r^{12}}{\left(r^{6}+R_{\mathrm{SS}}^{6}\right)\left(r^{6}+R_{\mathrm{ST}}^{6}\right.} .
$$

Eqn. (7) takes the radii $R_{\mathrm{ST}}$ and $R_{\mathrm{SS}}$ for Förster energy transfer of the processes (3a) and (3b) into account as well as the duration and shape of the excitation pulse in form of the parameter $p$, which can vary in the limits $p \approx 0$ and $p \approx 1$ for extremely short and long excitation pulses, respectively (see ref. 3). Parameter $p$ also depends on the lifetime of the first excited singlet state $\tau_{\mathrm{S}}=1 / k_{\mathrm{S}}$. The application of eqn. (7) for long-pulse excitation conditions will be central to this publication and further discussed below.

With the two modifications Smoluchowski's original and well known solution of eqn. (4),

$$
\rho(r, t)=1-\frac{R}{r} \operatorname{erfc}\left(\frac{r-R}{2 \sqrt{D t}}\right),
$$

as well as his famous result for the time-dependent rate coefficient $k_{2}$ for diffusion-controlled bimolecular reactions, ${ }^{1}$

$$
k_{2}(t)=4 \pi D R^{2}\left(\frac{\partial \rho(r, t)}{\partial r}\right)_{r=R}=4 \pi D R\left(1+\frac{R}{\sqrt{\pi D t}}\right)
$$

change in the case of diffusion-controlled TTA to

$$
\begin{aligned}
\rho_{\mathrm{A}}(r, t)=1 & -\frac{R_{\mathrm{A}}}{r} \operatorname{erfc}\left(\frac{r-R_{\mathrm{A}}}{2 \sqrt{D t}}\right) \\
& -\frac{1}{2 r \sqrt{\pi D t}} \int_{R_{\mathrm{A}}}^{\infty}\left[1-\rho_{\mathrm{A}}\left(r_{0}, 0\right)\right] r_{0}\left[\exp \left(-\frac{\left(r-r_{0}\right)^{2}}{4 D t}\right)\right. \\
& \left.-\exp \left(-\frac{\left(r+r_{0}-2 R_{\mathrm{A}}\right)^{2}}{4 D t}\right)\right] d r_{0}
\end{aligned}
$$

and

$$
k_{2 \mathrm{~A}}(t)=4 \pi D R_{\mathrm{A}}\left(1+\frac{R_{\mathrm{A}}}{\sqrt{\pi D t}}-f\left(\rho_{\mathrm{A}}(r, 0), R_{\mathrm{A}}, D, t\right)\right),
$$

with

$$
\begin{aligned}
f= & \frac{\pi}{2(\pi D t)^{3 / 2}} \int_{R_{\mathrm{A}}}^{\infty} r_{0}\left(r_{0}-R_{\mathrm{A}}\right)\left[1-\rho_{\mathrm{A}}\left(r_{0}, 0\right)\right] \\
& \times \exp \left(-\frac{\left(r_{0}-R_{\mathrm{A}}\right)^{2}}{4 D t}\right) \mathrm{d} r_{0},
\end{aligned}
$$

where $r_{0}>R_{\mathrm{A}}$ is the distance from the center of a reactive spherical surface of radius $R_{\mathrm{A}}$. The first two terms on the right side of eqn. (10) are identical with those of eqn. (8) for $R=R_{\mathrm{A}}$. The corresponding time-dependent rate coefficient $k_{2 \mathrm{~A}}(t)$ is also obtained from eqn. (9) with $R=R_{\mathrm{A}}$ and $\rho=\rho_{\mathrm{A}}$. The negative transient term $-f$ in eqn. (11a) is a consequence of using the new initial condition eqn. (7) as opposed to
Smoluchowski's initial condition eqn. (6). In an intermediate time range, $f$ is greater than the first transient term, $R_{\mathrm{A}} /$ $(\pi D t)^{-1 / 2}$; that means, instead of the rapid initial decrease of $k_{2}$ corresponding to Smoluchowski's initial condition, an initial increase of $k_{2 \mathrm{~A}}$ (so-called "anti-Smoluchowski time dependence" 4 ) is observed.

By measuring the time dependence of the delayed fluorescence of anthracene in highly viscous methylcyclohexaneethylcyclopentane (MCH-CP, 1:1 mixture) at temperatures between 105.0 and $117.5 \mathrm{~K}$ the above result was experimentally verified in ref. 3 . The analysis of the measurements, however, was limited by the following assumptions and/or experimental restrictions: (a) the first-order rate constant of the triplet decay, $k_{\mathrm{T}}$, which is needed in the evaluation procedure of the time dependence of the delayed fluorescence (see section 3 below) was taken from phosphorescence measurements and did not naturally emerge from the analysis procedure. (b) The relative diffusion coefficients $D=2 D_{0}$ of anthracene in $\mathrm{T}_{1}$ were estimated by extrapolating a room temperature value of the absolute diffusion coefficient $D_{0}$ of anthracene in $T_{1}$ in $\mathrm{MCH}$, assuming $D_{0} \propto T / \eta$ and using the known viscosities of $\mathrm{MCH} / \mathrm{MCP}$ at low temperatures. ${ }^{6}$ (c) The Förster radii $R_{\mathrm{SS}}$ and $R_{\mathrm{ST}}$ were assumed to be independent of the temperature in the range studied. (d) The analysis of the experiments in ref. 3 showed a dependence of $R_{\mathrm{ST}}$ and $R_{\mathrm{A}}$ on the time interval that was used for the evaluation procedure of the delayed fluorescence. (e) Although anthracene dissolves rather well in the glass-forming solvent $\mathrm{MCH}-\mathrm{MCP}$ at low temperatures, the long-term formation of micro-crystals in the sample, which significantly affect the measurements of the delayed fluorescence, could not be entirely avoided during long measurements (several hours).

The present investigation is aiming to remove these limitations through new measurements of the delayed fluorescence of anthracene in viscous solution (solvent mixture of cis-1,3and trans-1,4-dimethylcyclohexane) over a wider temperature range and upon excitation with a long laser pulse $(\sim 30 \mu \mathrm{s})$. The specific objectives are as follows:

(1) The validity of Smoluchowski's generalized theory is to be verified by measuring the delayed fluorescence and phosphorescence simultaneously for the same experimental conditions. The deviation of the rate constant of the triplet decay, $k_{\mathrm{T}}$, as obtained from independent analyses of both, delayed fluorescence and phosphorescence in a time range where Smoluchowski's theory is expected to hold ${ }^{4}$ will be used as a measure for its validity (section 4). This approach to test Smoluchowski's theory is possible since the fluorescence and phosphorescence spectra do not overlap in anthracene.

(2) Absolute values of $D$ are to be determined over a significant temperature range of the sample ${ }^{7-9}$ (sections 3 and 4). The temperature dependence of $D \propto T / \eta$ will be briefly discussed.

(3) TTA in eqn. (2) and the two FRETs in eqn. (3) are the three possible types of electronic energy transfer between molecules in the excited states $S_{1}$ or $T_{1}$. Reliable values of the characteristic radii $R_{\mathrm{A}}, R_{\mathrm{SS}}$ and $R_{\mathrm{ST}}$ are of fundamental photophysical interest. With the known values of $D$ the effective intermolecular annihilation distance $R_{\mathrm{A}}$ and the Förster radius $R_{\mathrm{ST}}$ can be determined. The temperature dependence of $R_{\mathrm{A}}$ and $R_{\mathrm{ST}}$ will yield information on the viscosity range in which Smoluchowski's equation is applicable.

(4) In a model by Butler and Pilling ${ }^{10}$ on the kinetics of TTA, Smoluchowski's boundary condition eqn. (5) was modified by introducing a first-order rate coefficient $k_{\mathrm{A}}(r)$ (denoted $l(r)$ in refs. 2, 10 and $k_{1 \mathrm{~A}}$ in 3) for the annihilation of a triplet pair, which decreases exponentially with increasing intermolecular distance $r$. This approach will be discussed on basis of our new data. In this context the parameters $A$ and $L$ characterizing the distance dependence of $k_{\mathrm{A}}(r)$ will be evaluated (section 4). 


\section{Experimental}

The choice of experimental conditions was essentially the same as in refs. 3 and 4 . The apparatus, however, has been entirely redesigned and setup from scratch. Its main components, schematically shown in Fig. 1, are outlined in the following together with the general measurement conditions:

\section{The substances}

Chromatographically treated anthracene was purified by vacuum sublimation and subsequent zone-refining. A new solvent mixture of $c i s-1,3$ - and trans-1,4-dimethylcyclohexane (ct$\mathrm{DMCH}$ ) was used. The solvents (Fluka, purum, >99\%) were individually purified by column chromatography on $\mathrm{Al}_{2} \mathrm{O}_{3}$ under nitrogen and mixed by volume at a $1: 1$ ratio at $-40^{\circ} \mathrm{C}$ according to their similar vapour pressure at this temperature. The mixture was dried over $\mathrm{K} / \mathrm{Na}$ and the anthracene solutions were finally degassed by 15 freeze-pump-thaw cycles. The anthracene concentration of $1.4 \times 10^{-5} \mathrm{M}$, which was lower than in previous measurements, was determined by absorption spectroscopy at room temperature. The choice of the solvent $c t$-DMCH as opposed to methylcyclohexanemethylcyclopentane (MCH-MCP) in ref. 3 is based on the following criteria:

(i) Anthracence exhibits a higher solubility in $c t-\mathrm{DMCH}$ than in $\mathrm{MCH} / \mathrm{MCP}$. Thus, the formation of anthracene microcystals at low temperatures, which may strongly disturb the delayed fluorescence (see ref. 11), is significantly reduced.

(ii) $c t$-DMCH forms an organic glass upon slow cooling and is photochemically very stable.

(iii) The appropriate range of viscosities of $c t-\mathrm{DMCH}$ for the present investigation is in a temperature range (132-150 $\mathrm{K}$ ), which is approximately $20 \mathrm{~K}$ higher compared to $\mathrm{MCH}-$ $\mathrm{MCP}$. Hence cooling times are shorter and thermalization as well as the long-term temperature stability are therefore expected to be improved. The temperature dependence of the viscosity of $c t-\mathrm{DMCH}$ was previously measured by standard capillary viscosimetry. ${ }^{12}$

\section{The excitation beam - generation of long excitation pulses}

The UV output of an argon ion laser at $363.3 \mathrm{~nm}$ (Spectra Physics, model 2045 , specified power of $\approx 3 \mathrm{~W}$ ), which is located in

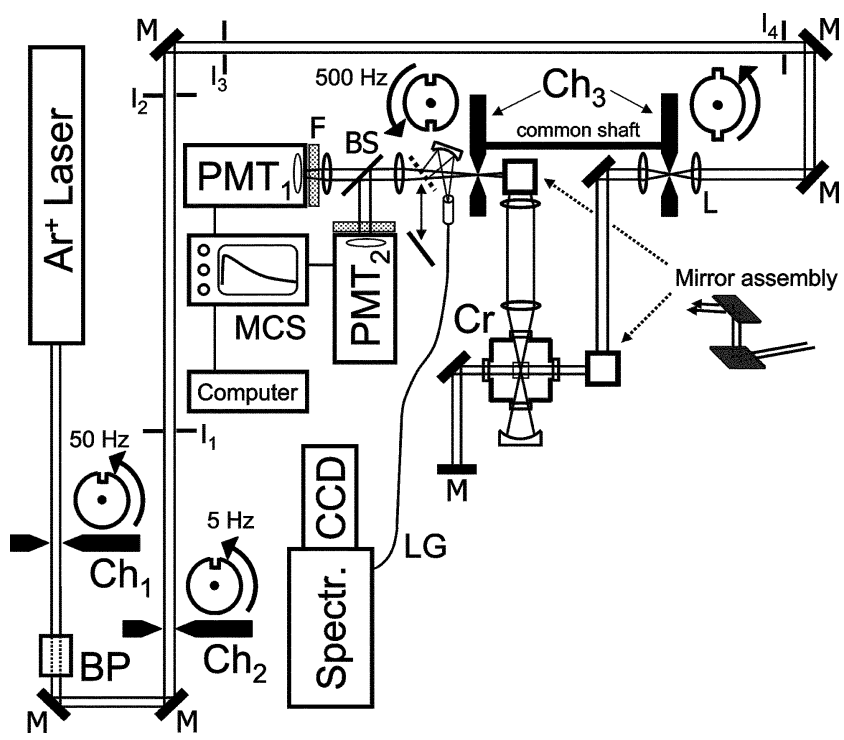

Fig. 1 Experimental setup schematically. AL: achromatic lens, BP: Brewster prism, BS: beam splitter, Ch: Chopper, Cr: cryostat, F: optical filter, $I_{k}(k=1-4)$ : irises, L: lens, LG: light guide, M: mirrors, MCS: multi channel scaler, PMT: photomultiplier. All major optical components were covered with light-tight cases and the excitation and detection beams were shielded with appropriate light-tight tubes, to reduce the amount of scattered light to a minimum. the origin of the $\mathrm{S}_{1} \leftarrow \mathrm{S}_{0}$ absorption band of anthracene (see Fig. 2), is used for the excitation of the sample. Long excitation pulses of $\approx 30 \mu$ s duration at a repetition rate of $\sim 5 \mathrm{~Hz}$ are generated by passing the $\mathrm{cw}$ beam through a set of three frequency-stabilized choppers whose disk-shapes and respective frequencies are shown in Fig. 1. $\mid$ At time intervals of $\sim 200$ ms (determined by the disk-shape and frequency of chopper 1) the three choppers are in phase (i.e. the light can pass all three choppers), leading to the exposure of the sample for approximately $\tau_{\mathrm{exc}} \approx 30 \mu \mathrm{s}$ (due to the disk-shape and frequency of chopper 3). In fact, chopper 3 consists of two disks on one common shaft, ${ }^{13}$ both disks are thus always in phase. By focussing the laser light onto the plane of disk 1 the cutoff time of chopper 3 is minimized and consequently the excitation pulse is rectangular in good approximation. Disk 2 is used on the detection side of the experiment (see Fig. 1) in order to strongly suppress the prompt fluorescence of anthracene at the time of excitation. The prompt emission of the sample, however, turned out to be so strong that light leaking through chopper 3 (disk 2) can be used to monitor the excitation pulse. The measured shape of the pulse is used to calculate the exact triplet concentration build-up for the analysis of the time dependence of the delayed fluorescence (see section 3).

In all experiments the excitation light is guided twice through the sample cell (10 $\mathrm{mm}$ pathlength), which is kept inside a home-built nitrogen cryostat. ${ }^{14}$ The laser beam is simply reflected back into the sample cell by a planar highly reflecting dielectric mirror which is placed $\sim 50 \mathrm{~cm}$ behind the cryostat (see Fig. 1). The second pass through the sample is to simply double the excitation efficiency. Due to the short coherence length of the UV laser light of $\sim 5 \mathrm{~cm}$ no interference occurs in the cell under these conditions.

The triplet concentration. With an extinction of $\varepsilon_{1 \leftarrow 0}(363$ $\mathrm{nm}) \approx 332 \mathrm{dm}^{3} \mathrm{~mol}^{-1} \mathrm{~cm}^{-1}$ at the excitation wavelength (see Fig. 2), $\Phi_{\mathrm{ISC}} \approx 0.5^{15}$ and the anthracene concentration of $\approx 1.4 \times 10^{-5} \mathrm{~mol} \mathrm{dm}{ }^{-3}$, an upper limit of the initial triplet concentration $\left[\mathrm{T}_{1}\right]_{0, \max }$ can be estimated to be $\leq 3.2 \times 10^{-9} \mathrm{~mol}$ $\mathrm{dm}^{-3}$ for an excitation time of $\tau_{\mathrm{exc}} \approx 30 \mu \mathrm{s}$ at a laser fluence of $\approx 450 \mu \mathrm{J} \mathrm{cm}^{-2}$ (laser power $3 \mathrm{~W}$, beam diameter $\approx 5 \mathrm{~mm}$ ). This value is smaller than in the preceding investigation. ${ }^{3}$

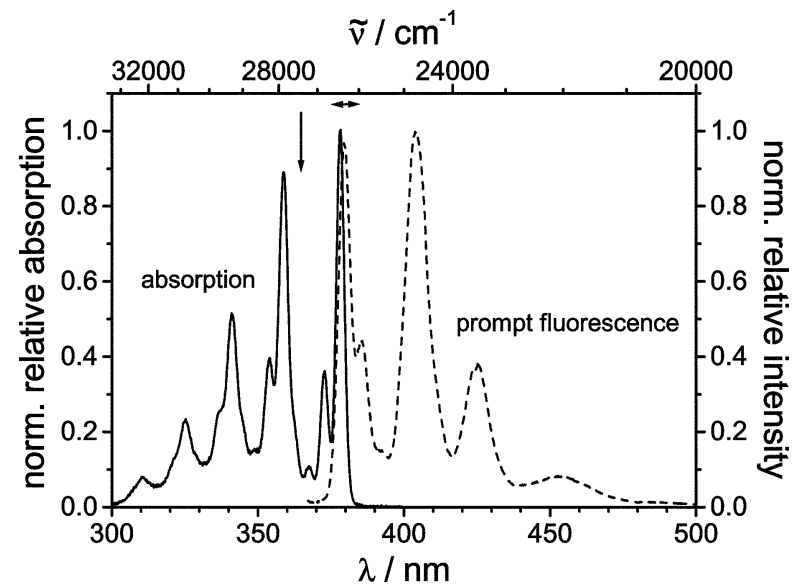

Fig. 2 Absorption spectrum of anthracene in $c t-\mathrm{DMCH}$ at room temperature (solid trace) and spectrum of the prompt fluorescence at $152 \mathrm{~K}$ (dashed). An interference filter at $377 \mathrm{~nm}$ (half-width $17 \mathrm{~nm}$ ) was used for the detection of the time dependence of the delayed fluorescence, the corresponding spectral detection region is indicated by means of a double arrow. The excitation wavelength is indicated by a vertical arrow.

|| The principle of creating long laser pulses from cw laser light is treated in detail in Fig. 6 of ref. 3. 


\section{The light detection}

The emission of the sample is collected with a spherical mirror and imaged with achromatic lenses via chopper 3 (disk 2) and a beam-splitter onto the photocathodes of two photomultipliers (Hamamatsu: R1464 - blue sensitive, 9125A - red sensitive), which are operated in photon counting mode (see Fig. 1). In all time-dependent measurements the photon counts are recorded with a multi-channel scaler (7882 Dual-InputMultiscaler, ComTech), operated at its minimum channel dwell time of $1.25 \mu \mathrm{s}$, over a total time interval of $81.92 \mathrm{~ms}$ (which is $\approx 2.3$ times longer than the triplet lifetime $\tau_{\mathrm{T}} \approx 35$ ms). Typical count rates varied between 400 and 4000 counts $\mathrm{s}^{-1}$ depending on the temperature. Accumulation times for decays were of the order of 10-13 h. The dead time of chopper 3 is $\approx 2.5 \mu$ s. A beam-splitter ( $>90 \%$ reflection $370-470 \mathrm{~nm}$, $>90 \%$ transmission $490-750 \mathrm{~nm}$ ) in the detection beam does not only spatially, but also spectrally separate the emission into wavelength regions corresponding to the (delayed) fluorescence and phosphorescence of anthracene. The final wavelength selection is achieved by means of a bandwidth filter ( $>50 \%$ transmission $370-380 \mathrm{~nm}$ ) in the detection path of the delayed fluorescence and a cut-off filter (RG665 - thickness $2 \mathrm{~mm}$, in the phosphorescence path). The corrected spectrum of the prompt fluorescence of anthracene in $c t-\mathrm{DMCH}$ is shown in Fig. 2 together with the corresponding absorption spectrum at room temperature.

\section{The measurement of spectra}

Emission spectra are measured using a spectrograph (Chromex, 500IS/SM) in combination with a CCD camera (Photometrics, SDS 9000). In Fig. 1 the position in the setup is indicated, where the prompt fluorescence (PF), the delayed fluorescence (DF) or the phosphorescence (PH) can be collected with a broad-band light-guide, which is connected to the entrance slit of the spectrograph. The accumulation time for the prompt fluorescence spectrum was $\approx 1 \mathrm{~min}$. A calibration lamp was used to correct for the spectral intensity dependence of the light guide, the spectrograph and the CCD-camera.

\section{The cooling of the sample}

The sample was placed into a home-built (liquid $\mathrm{N}_{2}$ ) cryostat and its temperature was controlled with an auto-tuning PID temperature controller (Model 330, Lake Shore Cryotronics). The temperature was measured with an absolute accuracy of $\pm 0.1 \mathrm{~K}$ using a calibrated Pt-100 resistor (Pt-103-14L). The constancy of the temperature was better than $\pm 0.05 \mathrm{~K}$ over the duration of a measurement of the delayed fluorescence.

\section{The time dependence of the delayed fluorescence}

The time dependence of the delayed fluorescence $I_{\mathrm{DF}}$ can be directly obtained from the time dependence of $k_{2 \mathrm{~A}}$ for appropriately chosen experimental conditions, based on the kinetic model introduced above (see also ref. 3). As long as the initial triplet concentration $\left[T_{1}\right]_{0}$ is sufficiently small, the contribution of TTA to the total triplet decay is insignificant, i.e. $k_{\mathrm{T}} \gg k_{2 \mathrm{~A}}(\infty)\left[\mathrm{T}_{1}\right]_{0}$, and the time-dependent intensity of the delayed fluorescence can be written in the form

$$
I_{\mathrm{DF}}(t)=C k_{2 \mathrm{~A}}(t)\left[\mathrm{T}_{1}\right]_{0}^{2} \exp \left(-2 k_{\mathrm{T}} t\right),
$$

where $k_{2 \mathrm{~A}}(t)$ is given by eqn. (11) and $C=C\left(\Phi_{\mathrm{S}}\right)$ is an experimental factor that depends on the fluorescence quantum yield $\Phi_{\mathrm{S}}$ (among other variables). The most important aspects to determine all relevant kinetic quantities by numerically calculating $k_{2 \mathrm{~A}}(t)$ and fitting eqn. (12) to measured curves of the time-dependent delayed fluorescence will be described in the following. The objective is to determine (i) the effective annihilation radius $R_{\mathrm{A}}$ and the relative diffusion coefficient $D$ of anthracene in $\mathrm{T}_{1}$, which are associated with Smoluchowski's original model, (ii) the Förster radius $R_{\mathrm{ST}}$ in the initial condition eqn. (7), which governs the anti-Smoluchowski region of $I_{\mathrm{DF}}(t)^{4}\left(R_{\mathrm{SS}}\right.$ cannot be determined in the case of long-pulse excitation, see below) and finally (iii) the triplet decay rate coefficient $k_{\mathrm{T}}$ :

For excitation with a long laser pulse, as in the present case (see eqn. (7)), the time $t=0$ is not well defined and the generation of triplet pairs during the excitation process has to be taken into account. In ref. 3 the exact shape of the excitation pulse was not known from the experiments, however, a proper analysis of $I_{\mathrm{DF}}(t)$ including the build-up of the triplet concentration during excitation was described by eqns. (36)-(39) in ref. 3. The corresponding formalism is applied in this publication using the measured temporal quasi-rectangular profile of the laser pulse (the inset in Fig. 4 shows the rectangular excitation profile during the first chopper interrupt (homogeneous excitation), which was monitored by the fraction of the strong prompt fluorescence (PF) that leaked through chopper 3 towards the detector). From the excitation duration $\tau_{\mathrm{exc}} \approx$ $30 \mu \mathrm{s}$ and the lifetime** $\tau_{\mathrm{S}}=6 \pm 1$ ns of $\mathrm{S}_{1}$ the parameter $p$ in the initial condition (eqn. (7)) can be calculated: ${ }^{3}$ $p=1-\left(2 \tau_{\mathrm{S}} / \tau_{\mathrm{exc}}\right)+2\left(\tau_{\mathrm{S}} / \tau_{\mathrm{exc}}\right)^{2}\left[1-\exp \left(-\tau_{\mathrm{exc}} / \tau_{\mathrm{S}}\right)\right]=0.9995 . \mathrm{A}$ change of $\pm 1 \mathrm{~ns}$ in $\tau_{\mathrm{S}}$ would correspond to a deviation of $<1 \times 10^{-4}$ in $p$. Therefore the measurement error of $\tau_{\mathrm{S}}$ and a possible slight decrease of the singlet lifetime over the temperature region of interest ${ }^{16}$ are deemed negligible in this study. The value of $p$ is so close to unity, i.e. to the ultimate long-pulse excitation limit, that the second term in the initial condition, $\rho_{\mathrm{A}}(r, 0)$, is virtually zero and thus the Förster radius, $R_{\mathrm{SS}}$, of $\mathrm{S}_{1}-\mathrm{S}_{1}$ energy transfer is in fact irrelevant in the evaluation procedure of $I_{\mathrm{DF}}(t)$ and cannot be deduced. This is a consequence of the fact that $S_{1} \cdots S_{1}$ pairs are never precursors of $\mathrm{T}_{1} \cdots \mathrm{T}_{1}$ pairs in the limit of longpulse excitation; $S_{1}-S_{1}$ energy transfer is therefore entirely negligible in the present experiments.

In order to determine absolute values of the radii $R_{\mathrm{A}}, R_{\mathrm{ST}}$ and the triplet decay $k_{\mathrm{T}}$ by fitting eqn. (12) to an experimental curve $I_{\mathrm{DF}}(t)$, a simplex variation ${ }^{17}$ was used, in which the weighted sum of the squared deviations (least square error) was minimized. The proportionality constant $C^{\prime} \equiv C\left(\Phi_{\mathrm{S}}\right)\left[\mathrm{T}_{1}\right]_{0}^{2}$ in eqn. (12) was simply calculated in the minimization of the least square error. For this approach the value of the diffusion coefficient $D$ of molecules in the triplet state must be known for a given temperature. Known values of the absolute diffusion coefficient ${ }^{9} D_{0}=D / 2$ for a similar solvent at room temperature were used in calculations in previous work ${ }^{3,4}$ on TTA. In refs. 3 and $4, D_{0}$ values were extrapolated with the Stokes-Einstein equation to relevant low temperatures and high viscosities, with an uncertainty of at least $\pm 25 \%$. In order to remove this uncertainty, $D_{0}$ must be determined from measurements of $I_{\mathrm{DF}}(t)$ taken under the same experimental conditions. At first glance one would expect that $D_{0}$ could simply be obtained in the simplex fit of eqn. (12) to the measured time dependence of the delayed fluorescence, if all relevant quantities used in the Smoluchowski model, i.e. $D=2 D_{0}, R_{\mathrm{A}}, R_{\mathrm{ST}}$ and $k_{\mathrm{T}}$, are treated as free parameters (at a given temperature for $p \approx 1)$. However, since $k_{2 \mathrm{~A}}(t)$ is a function of the ratio $R_{\mathrm{A}}^{2} / D$ and the parameters $D$ and $R_{\mathrm{A}}$ never occur individually in eqn. (11), there is a very strong correlation of $D$ and $R_{\mathrm{A}}$. One way of eliminating this strong $D \leftrightarrow R_{\mathrm{A}}$ correlation is to determine relative diffusion coefficients in measurements with a modified excitation scheme. Upon spatially periodic

** The value of $\tau_{\mathrm{S}}$ was measured in a separate experiment using a short-pulse $\mathrm{N}_{2}$-laser pumped dye laser, using a fast photodiode (rise-time $<1 \mathrm{~ns}$ ) and oscilloscope (Tektronix, TDS 3052, $500 \mathrm{MHz}$ ). 
excitation (in the following denoted with the acronym 'per') of the sample with two interfering laser beams, as opposed to conventional homogeneous excitation (abbreviated 'hom') measurements, a spatially-periodic distribution of triplet state molecules is generated. ${ }^{7}$ The evaluation procedure to determine $D$ from the time dependence of $I_{\mathrm{DF}}$ under these conditions, which essentially minimizes the extent to which $D$ and $R_{\mathrm{A}}$ correlate, is based on the fact that diffusion only affects the amplitude of the deviation from the average spatial triplet concentration. The published analysis procedure (ref. 9, section 2.1) requires an adequate modification in the present case for the following reason: According to Smoluchowski, the relative spatial distribution of triplet state molecules will change due to TTA itself. The time-dependent local distribution $\rho_{\mathrm{A}}(r, t)$ of triplets in the neighbourhood of a selected triplet molecule deviates significantly from the average spatial concentration of triplets. Even in the stationary state, where the relative concentration is $\rho_{\mathrm{A}}(r, \infty)=1-R_{\mathrm{A}} / r$ according to Smoluchowski, the deviation from the spatial average is still significant at $r=10 R_{\mathrm{A}}$. This effect becomes only negligible for large spatial periods $d \geq 1000 R_{\mathrm{A}}$. Hence, due to the temporal change of $\rho_{\mathrm{A}}(r, t)$ in Smoluchowski's theory the time-dependent rate coefficient $k_{2 \mathrm{~A}}(t)$ should be used in case of spatially periodic excitation, rather than the inadequate stationary value $k_{2 \mathrm{~A}}(\infty)$ used in 9 (see assumption (d) on page 506 in ref. 9). In the following a modified expression for $k_{2 \mathrm{~A}}(t)$ (see eqn. (11)) will be derived for the case of spatially periodic excitation, which can be used to determine absolute values of $D_{0}$ by fitting eqn. (12) to the measured time dependence of the delayed fluorescence.

\section{Spatially periodic excitation}

We consider the excitation of a sample with a standing wave of period $d$, which in practice is based on the incomplete interference of two laser beams with a phase shift $\theta$, propagating along a defined direction $z$. The arising periodic concentration $c_{\mathrm{T}}(z)$ of triplet molecules under these excitation conditions is given in a normalized form by

$$
c_{\mathrm{T}}(z)=1+b \cos \left(\frac{2 \pi(z-\theta)}{d}\right)
$$

as graphically shown in Fig. 3. The incomplete interference is accounted for by the parameter $b$, which can take values between 0 (no interference), corresponding to spatially entirely homogeneous excitation, and 1 (full interference - normalized), corresponding to spatially entirely periodic excitation. The average normalized concentration $c_{\mathrm{T}}^{\text {per }}(r)$ of triplet molecules at distance $r$ has to be derived from eqn. (13) using the initial condition in eqn. (7). For that purpose we consider a triplet molecule in an arbitrary position relative to the periodic interference pattern, which corresponds to an arbitrary value of the phase difference in the range $0 \leq \theta \leq d$. Assuming the selected triplet molecule to be in the origin of a spherical coordinate system, the sought relative concentration $c_{\mathrm{T}}^{\text {per }}(r)$ at distance $r$ can be found by integration of $c_{\mathrm{T}}(z)$ over a sphere of radius $r$ around the selected triplet molecule and subsequent averaging over all phases $\theta$ within one period $d$, allowing for the relative probabilities for different values of $\theta$ to occur. The resulting distribution is given by

$$
c_{\mathrm{T}}^{\mathrm{per}}(r)=1+\frac{b^{2} d}{4 \pi r} \sin \left(\frac{2 \pi r}{d}\right)
$$

an example of $c_{\mathrm{T}}^{\text {per }}(r)$ is shown in Fig. 3. For an adequate modification of the second-order rate constant $k_{2 \mathrm{~A}}(t)$ which takes the spatially periodic excitation into account $\rho_{\mathrm{A}}(r, 0)$ in eqn. (11b) must simply be multiplied by $c_{\mathrm{T}}^{\text {per }}(r)$. The integral in eqn. (11b) can then be solved analytically in the case of the original Smoluchowski initial condition eqn. (6) and an (a)

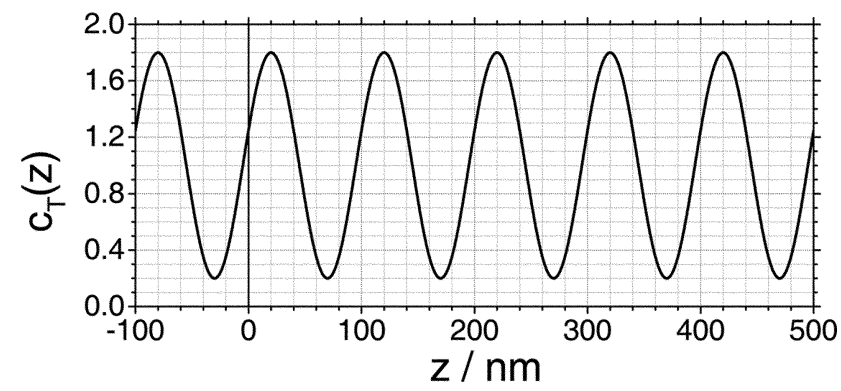

(b)

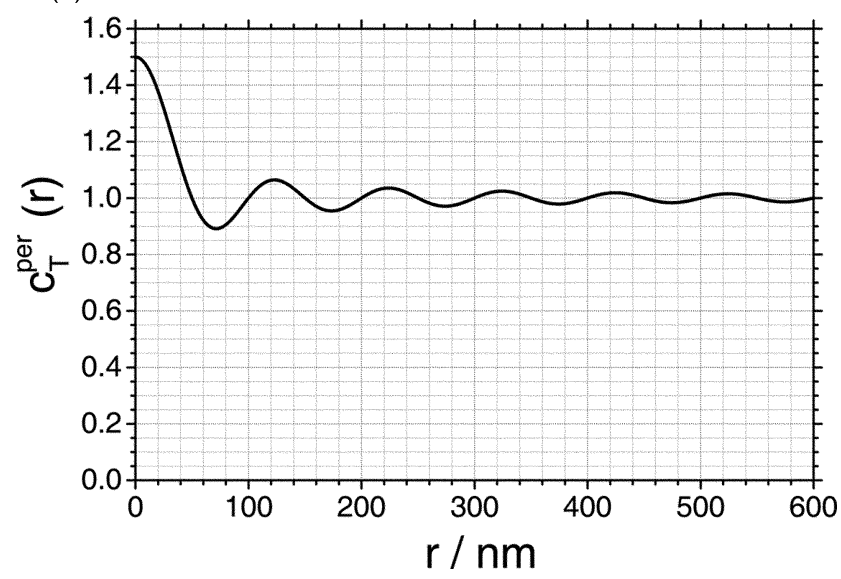

Fig. 3 (a) Illustration of spatially periodic excitation $\left(c_{\mathrm{T}} v s . z\right)$ with period $d$, phase shift $\theta$ and a fraction $b=0.8$ of a spatially homogeneous background according to eqn. (13). Parameter values: $d=100$ $\mathrm{nm}, \theta=20 \mathrm{~nm}, b=0.8$. (b) Average relative concentration $c_{\mathrm{T}}^{\text {per }}$ of triplet molecules at a distance $r$ from a selected triplet molecule upon spatially periodic excitation according to eqn. (14). Parameter values: $d=100 \mathrm{~nm}, b=1$.

explicit expression for $k_{2 \mathrm{~A}}(t)$ can be derived assuming $d \gg R_{\mathrm{A}}: \dagger \dagger$

$$
\begin{aligned}
k_{2 \mathrm{~A}}(t)= & 4 \pi D R_{\mathrm{A}}\left[1+\frac{R_{\mathrm{A}}}{\sqrt{\pi D t}}\right. \\
& \left.+\frac{b^{2}}{2}\left(\frac{R_{\mathrm{A}}}{\sqrt{\pi D t}}+\exp \left(-\left(\frac{2 \pi}{d}\right)^{2} D t\right)\right)\right]
\end{aligned}
$$

Eqn. (15) is valid to describe the time dependence of the delayed fluorescence in a time interval which is governed by the original Smoluchowski behaviour, i.e. in the tail of $I_{\mathrm{DF}}(t)$. If the delayed fluorescence in the time range governed by anti-Smoluchowski behaviour is considered, ${ }^{4}$ the modified boundary condition $\rho_{\mathrm{A}}(r, 0)$ in eqn. (7) must be applied and the factor $f$ in eqn. (11) must be taken into account. In this case

$$
k_{2 \mathrm{~A}}(t)=4 \pi D R_{\mathrm{A}}\left[\gamma_{2 \mathrm{~A}}^{\mathrm{hom}}(t)+\gamma_{2 \mathrm{~A}}^{\mathrm{per}}(t)\right], \text { with }
$$

t† Eqn. (15) is an approximate solution of eqn. (11) for spatially periodic excitation in case of Smoluchowski's original initial condition eqn. (6). The exact form is given by: $k_{2 \mathrm{~A}}(t)=$ $4 \pi D R_{\mathrm{A}}\left(1+A_{1}+A_{2}+A_{3}\right)$, with

$$
\begin{aligned}
& A_{1}=\frac{R_{\mathrm{A}}}{\sqrt{\pi D t}}\left(1+\frac{b^{2}}{2} \frac{\sin \left(2 \pi R_{\mathrm{A}} / d\right)}{2 \pi r / d}\right), \\
& A_{2}=\frac{b^{2}}{2} \cos \left(\frac{2 \pi R_{\mathrm{A}}}{d}\right) \exp \left(-\left(\frac{2 \pi}{d}\right)^{2} D t\right), \\
& A_{3}=-\frac{b^{2}}{\sqrt{\pi}} \sin \left(\frac{2 \pi R_{A}}{d}\right) \int_{0}^{\infty} \sin \left(\frac{4 \pi \sqrt{D t} x}{d}\right) \exp \left(-x^{2}\right) \mathrm{d} x .
\end{aligned}
$$




$$
\begin{aligned}
\gamma_{2 \mathrm{~A}}^{\text {hom }}(t)= & 1+\frac{R_{\mathrm{A}}}{\sqrt{\pi D t}}-\frac{\pi}{2(\pi D t)^{3 / 2}} \int_{R_{\mathrm{A}}}^{\infty} r_{0}\left(r_{0}-R_{\mathrm{A}}\right) \\
& \times\left[1-\rho_{\mathrm{A}}\left(r_{0}, 0\right)\right] \exp \left(-\frac{\left(r_{0}-R_{\mathrm{A}}\right)^{2}}{4 D t}\right) \mathrm{d} r_{0}
\end{aligned}
$$

and

$$
\begin{aligned}
\gamma_{2 \mathrm{~A}}^{p e r}(t)= & +\frac{\pi}{2(\pi D t)^{3 / 2}} \int_{R_{\mathrm{A}}}^{\infty} r_{0}\left(r_{0}-R_{\mathrm{A}}\right) \rho_{\mathrm{A}}\left(r_{0}, 0\right) \frac{b^{2} d}{4 \pi r} \\
& \times \sin \left(\frac{2 \pi r}{d}\right) \exp \left(-\frac{\left(r_{0}-R_{\mathrm{A}}\right)^{2}}{4 D t}\right) \mathrm{d} r_{0},
\end{aligned}
$$

where $\gamma_{2 \mathrm{~A}}^{\text {hom }}(t)$ and $\gamma_{2 \mathrm{~A}}^{\text {per }}(t)$ represent the time-dependent parts of $k_{2 \mathrm{~A}}(t)$ resulting from spatially homogeneous and periodic excitation respectively. Eqn. (16) cannot be calculated analytically and the integrals must be evaluated numerically. This approach was taken by us for the analysis of delayed fluorescence data, which were measured upon spatially periodic excitation. The experimental results obtained upon spatially periodic excitation, however, were not as satisfactory as expected for the following reasons:

(A) Different from the method used in ref. 9, a standing wave inside the sample was created by back-reflecting the laser beam off the wall of an especially coated cuvette, which is highly reflecting at $363 \mathrm{~nm}$ on one side. Considering the coherence length of $\sim 5 \mathrm{~cm}$ of the laser light, it was expected to generate an interference pattern in the form of a standing wave with a spatial period of $d=\lambda(2 n)^{-1}$ under these conditions; $n(\lambda, T)$ is the refractive index of the solvent at $\lambda=363 \mathrm{~nm}$ in the respective temperature range, which is accurately known from temperature dependent measurements published elsewhere. ${ }^{18}$ Although the interference cannot be expected to be complete, it turned out, that the (fit) parameter $b$ in eqn. (16b) was particularly low, varying between $\approx 0$ and 0.3 $(\sim 4.5 \%$ effect $)$. Since in approximately half of the measurements interference effects of less than $\sim 5 \%$ were obtained the temperature dependent results are not as reliable as previously expected. The problem of an insufficient interference could not be overcome experimentally, even when an experimental arrangement such as in ref. 9 was used. Although the interference effect was improved to $\approx 30 \%$ by this approach (theoretically $50 \%$ effects are possible upon full interference - see eqn. (15) for $b=1)$, the detection setup was not designed for that particular excitation geometry and the measured time dependence of the delayed fluorescence was very noisy and hence the analysis results were again severely affected.

(B) With the known refractive index of $c t$ - DMCH at low temperatures (see ref. 18) the spatial period $d$ was calculated to be $\approx 119 \mathrm{~nm}$, which is a factor of $\sim 100$ larger than $R_{\mathrm{A}}$, and hence may not be sufficient to entirely satisfy the criterion $d \gg R_{\mathrm{A}}$.

In conclusion, if a sufficient interference effect can be achieved together with a very good signal-to-noise ratio in measurements of the delayed fluorescence, the outlined procedure should yield values of $D_{0}$ of anthracene in $\mathrm{T}_{1}$ with an accuracy better than $\pm 10 \%$.

\section{Results and discussion}

In all time-dependent measurements the emission was measured for $81.9 \mathrm{~ms}$ with a step size of $1.28 \mu \mathrm{s}$ ( $\sim 65000$ datapoints). All data were rate-corrected, and dark counts were subtracted from the measured luminescences. Data-points that were recorded at times when the detection beam was disrupted by chopper 3 were removed, and the number of points per curve was further reduced by summing over ten adjacent data-points. In that way the signal-to-noise ratio was increased from $\approx 25$ to $\approx 125$ in case of the delayed fluorescence. Typical measurements of the time-dependent delayed fluorescence, $I_{\mathrm{DF}}$, of anthracene in $c t$-DMCH at $136 \mathrm{~K}$ are shown in Fig. 4 for spatially homogeneous $\left(I_{\mathrm{DF}(\mathrm{hom})}\right)$, and spatially periodic $\left(I_{\mathrm{DF}(\mathrm{per})}\right)$ excitation of the sample. $I_{\mathrm{DF} \text { (per) }}$ was normalized to the value of $I_{\mathrm{DF}(\mathrm{hom})}$ at $10 \mathrm{~ms}$ (vertical arrow in Fig. 4) in order to demonstrate the different emission response for the two excitation conditions. Spatially periodic excitation resulted in a $3.5 \%$ effect in the present case. The mean deviation of the corresponding fit parameters, which are listed for $I_{\mathrm{DF}(\mathrm{per})}$ and $I_{\mathrm{DF}(\mathrm{hom})}$ in the figure caption, is less than $2 \%$ (also see Tables 1 and 2). The time axis in Fig. 4 is displayed logarithmically in order to illustrate the transition from the initial antiSmoluchowski behaviour of the time dependence, where $k_{2 \mathrm{~A}}(0) / k_{2 \mathrm{~A}}(\infty) \ll 1$ (the initial intensity increase ${ }^{4}$ ), to the part of the curve, which is entirely governed by Smoluchowski temporal behaviour, characterized by $k_{2 \mathrm{~A}}(0) / k_{2 \mathrm{~A}}(\infty) \gg 1$ (the tail of the curve); see refs. 3 and 4 . The transition from the antiSmoluchowski to the Smoluchowski temporal behaviour of $I_{\mathrm{DF}}$ is fully accounted for by $k_{2 \mathrm{~A}}(t)$ according to eqn. (11), which is based on the modified initial condition for the relative concentration of reactive particles $\rho_{\mathrm{A}}(r, 0)$ in eqn. (7). The fact that "flawless" anti-Smoluchowski behaviour is observed indicates that disturbing emissions due to anthracene microcrystals

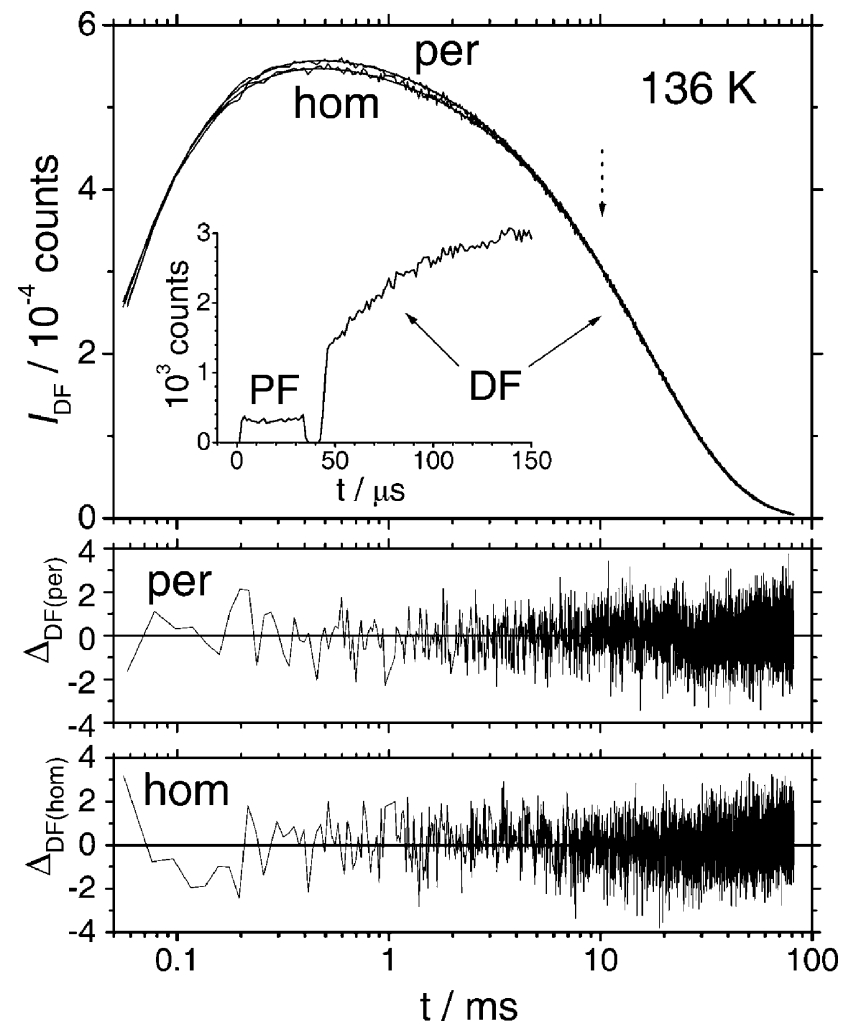

Fig. 4 Semi-logarithmic plot of the time dependence of the delayed fluorescence (DF) of anthracene in $c t-\mathrm{DMCH}$ at $136 \mathrm{~K}$ upon spatially periodic (per) and homogeneous (hom) excitation. Upper graph: measured intensities, $I_{\mathrm{DF} \text { (per) }}$ (upper trace) and $I_{\mathrm{DF}(\mathrm{hom})}$ (lower trace), as well as the calculated intensity values $I_{\mathrm{DF}, \mathrm{cal}}$, for each curve. Fit parameters used in eqn. (12) - per: $k_{\mathrm{T}, \mathrm{DF}}=28.90 \mathrm{~s}^{-1}, D_{0}=22795 \mathrm{~nm}^{2} \mathrm{~s}^{-1}$, $R_{\mathrm{A}}=0.92 \mathrm{~nm}, R_{\mathrm{ST}}=4.13 \mathrm{~nm}$; hom: $k_{\mathrm{T}, \mathrm{DF}}=28.91 \mathrm{~s}^{-1}, D_{0}=22237$ $\left.\mathrm{nm}^{2} \mathrm{~s}^{-1}, R_{\mathrm{A}}=0.95 \mathrm{~nm}, R_{\mathrm{ST}}=4.08 \mathrm{~nm}\right) . I_{\mathrm{DF}(\mathrm{hom})}$ was normalized to $I_{\mathrm{DF} \text { (per) }}$ at times larger than $10 \mathrm{~ms}$ indicated by the dotted vertical arrow. Lower graphs: weighted residuals $\Delta_{\mathrm{DF}}=\left(I_{\mathrm{DF}}-I_{\mathrm{DF}, \mathrm{cal}}\right) / \frac{1}{I_{\mathrm{DF}}^{2}}$ for fits of measurements 'per' and 'hom'. The interrupts created by chopper 3 have been eliminated from the plots. Insert upper graph: First $200 \mu$ s of the DF(hom) in a linear plot (first chopper interrupt). The signal in the first $\sim 30 \mu$ s is due to strong prompt fluorescence (PF) leaking along the detection beam during closure of chopper 3 . 
Table 1 Parameters anthracene in $c t$-DMCH; $T$ : temperature; $\eta$ : viscosity of $c t$-DMCH; ${ }^{12} D_{\mathrm{SE}}$ : absolute diffusion coefficient of triplet anthracene calculated with eqn. (17) and $r=0.16 \mathrm{~nm}$, based on spatially periodic excitation. $D_{0}$ : absolute diffusion coefficient of triplet anthracene. $R_{\mathrm{A}}$ : effective annihilation radius. $k_{2 \mathrm{~A}}^{\mathrm{m}}(\infty)$ : steady-state rate coefficient for TTA according to the modified Smoluchowski model $(\mathrm{m}=$ Smol) and obtained from the empirical parameters $A$ and $L$ according to Butler and Pilling $(\mathrm{m}=\mathrm{BP})$. O: Obtained through fit with $D_{\mathrm{SE}}$ fixed (weighting applied), $\bullet$ : fit with weighting procedure, $*$ : fit without weighting procedure. Förster radius for singlet-singlet energy transfer $R_{\mathrm{SS}}=5.0 \mathrm{~nm}$ for all data. All measurements are in the long-pulse excitation limit $\mathrm{p}=0.9995$ (see initial condition (7) and eqn. (24) in ref. 3)

\begin{tabular}{|c|c|c|c|c|c|c|c|c|c|c|}
\hline$T / \mathrm{K}$ & $T \eta^{-1} / \mathrm{K} \mu \mathrm{Pa}^{-1} \mathrm{~s}^{-1}$ & $D_{0}^{\bullet} / \mathrm{nm}^{2} \mathrm{~s}^{-1}$ & $D_{\mathrm{SE}}{ }^{\circ} / \mathrm{nm}^{2} \mathrm{~s}^{-1}$ & $D_{0} * / \mathrm{nm}^{2} \mathrm{~s}^{-1}$ & $D_{0}^{\bullet} / D_{0}^{\text {fit }}$ & $R_{\mathrm{A}} \bullet / \mathrm{nm}$ & $R_{\mathrm{A}}{ }^{\circ} / \mathrm{nm}$ & $R_{\mathrm{A}} * / \mathrm{nm}$ & $\begin{array}{l}k_{2 \mathrm{~A}}^{\mathrm{Smol}}(\infty) / \\
10^{-5} \mathrm{~s}^{-1}\end{array}$ & $\begin{array}{l}k_{2 \mathrm{~A}}^{\mathrm{BP}}(\infty) / \\
\left.10^{-5} \mathrm{~s}^{-1}\right]\end{array}$ \\
\hline 134 & 2.57 & 11937 & 11787 & 10775 & 1.207 & 0.90 & 0.89 & 1.18 & 2.69 & 3.39 \\
\hline 135 & 3.60 & 16821 & 16511 & 15612 & 1.216 & 0.87 & 0.86 & 1.08 & 3.67 & 4.67 \\
\hline 136 & 4.97 & 22237 & 22795 & 21208 & 1.165 & 0.95 & 0.96 & 1.07 & 5.31 & 6.07 \\
\hline 137 & 6.76 & 30478 & 31005 & 28603 & 1.174 & 0.91 & 0.92 & 1.04 & 6.95 & 8.14 \\
\hline 138 & 9.08 & 38091 & 41645 & 37598 & 1.092 & 0.99 & 1.04 & 1.05 & 9.50 & 10.02 \\
\hline 139 & 12.05 & 49623 & 55267 & 49920 & 1.072 & 1.04 & 1.10 & 1.03 & 12.99 & 12.82 \\
\hline 140 & 15.79 & 63161 & 72421 & 64148 & 1.041 & 1.03 & 1.10 & 1.03 & 16.30 & 16.04 \\
\hline 141 & 20.48 & 83471 & 93931 & 83651 & 1.061 & 0.99 & 1.03 & 0.99 & 20.67 & 20.78 \\
\hline 142 & 26.28 & 105415 & 120533 & 107957 & 1.044 & 0.93 & 0.99 & 0.96 & 24.51 & 25.80 \\
\hline 143 & 33.39 & 125899 & 153143 & 134748 & 0.982 & 0.98 & 1.08 & 0.10 & 30.97 & 30.41 \\
\hline 144 & 42.04 & 152164 & 192816 & 174549 & 0.942 & 0.96 & 1.07 & 1.01 & 36.78 & 36.23 \\
\hline 145 & 52.45 & 196632 & 240562 & 238243 & 0.976 & 0.93 & 1.03 & 1.03 & 45.72 & 45.90 \\
\hline 146 & 64.90 & 246251 & 297663 & 366788 & 0.988 & 0.90 & 0.99 & 1.13 & 55.62 & 56.48 \\
\hline 147 & 79.67 & 283344 & 365406 & 487461 & 0.926 & 0.91 & 1.03 & 1.20 & 64.66 & 64.27 \\
\hline 148 & 97.08 & 344893 & 445257 & 672035 & 0.925 & 0.89 & 1.01 & 1.29 & 76.97 & 77.01 \\
\hline 149 & 117.47 & 449026 & 538775 & 928430 & 0.995 & 0.88 & 0.97 & 1.40 & 99.78 & 98.14 \\
\hline 150 & 141.19 & 580050 & 647565 & 12667303 & 1.070 & 0.91 & 0.96 & 1.51 & 132.75 & 124.15 \\
\hline
\end{tabular}

are not present and that recombination fluorescence based on UV multi-photon ionization is negligible, as expected in the case of long-pulse excitation.

The upper graph in Fig. 4 also shows the calculated intensities, $I_{\mathrm{DF} \text { (cal) }}$, which result from a fit of eqn. (12) to the respective measured curves applying eqn. (16) for the calculation of $k_{2 \mathrm{~A}}(t)$ with appropriate parameters for the respective excitation scheme. The lower part of Fig. 4 shows the weighted residuals, $\Delta_{\mathrm{DF}(k)}=\left(I_{\mathrm{DF}(k)}-I_{\mathrm{DF}(\mathrm{cal})}\right) / \frac{1}{I_{\mathrm{DF}(k)}}(k=$ hom, per $)$ of the fits to demonstrate the quality of the least square minimization. The new custom-designed fitting program for eqn. (12) takes all aspects of the modified Smoluchowski model for TTA into account and therefore surpasses previous analysis procedures in several ways, which are briefly outlined in the following:

(a) In the present study the relative diffusion coefficient $D$ at $136 \mathrm{~K}$ (Fig. 4) follows from the analysis of $I_{\mathrm{DF} \text { (per) }}$. Its value was determined with an uncertainty of approximately $\pm 10 \%$ and estimates of start parameters $D(t)$ for fits are based on this value. This represents an improvement in comparison to refs. 3 and 4 where room-temperature values of $D$ for anthracene in methylcyclohexane $^{9}$ were extrapolated with the StokesEinstein equation to the temperature region of interest. Due to this extrapolation and the fact that the diffusion coefficient was only known for a similar solvent, the uncertainty of $D$ used to be at least $\pm 25 \%$.

(b) In the present work $R_{\mathrm{ST}}$ is a variable parameter for all temperatures and follows from the analysis of $I_{\mathrm{DF}}(t)$. In ref. 3 the Förster radius $R_{\mathrm{ST}}$ was deduced to be $4.2 \mathrm{~nm}$ from the anti-Smoluchowski behaviour at only one particular temperature and then assumed to be temperature-independent in the temperature range of interest.

(c) The initial part of the delayed fluorescence, which is governed by an anti-Smoluchowski time dependence, is very short

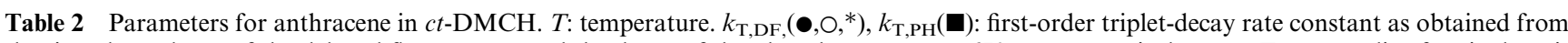
the time dependence of the delayed fluorescence and the decay of the phosphorescence at 670 nm, respectively. $R_{\mathrm{ST}}:$ Förster radius for singlet-triplet energy transfer; o: obtained through fit with $D_{\mathrm{SE}}$ fixed (weighting applied), $\bullet$ : fit with weighting procedure, *: fit without weighting procedure. Förster radius for singlet-singlet energy transfer $R_{\mathrm{SS}}=5.0 \mathrm{~nm}$ for all data. All measurements are in the long-pulse excitation limit $p=0.9995$ (see initial condition (7) and eqn. (24) in ref. 3)

\begin{tabular}{|c|c|c|c|c|c|c|c|c|}
\hline$T / \mathrm{K}$ & $T^{-1} / \mathrm{K}^{-1}$ & $k_{\mathrm{T}, \mathrm{DF}} \bullet / \mathrm{s}^{-1}$ & $k_{\mathrm{T}, \mathrm{DF}}{ }^{\circ} / \mathrm{s}^{-1}$ & $k_{\mathrm{T}, \mathrm{DF}}{ }^{*} / \mathrm{s}^{-1}$ & $k_{\mathrm{T}, \mathrm{PH}} / \mathrm{s}^{-1}$ & $R_{\mathrm{ST}} \bullet / \mathrm{nm}$ & $R_{\mathrm{ST}}{ }^{\circ} / \mathrm{nm}$ & $R_{\mathrm{ST}} * / \mathrm{nm}$ \\
\hline 134 & 52140 & 28.86 & 28.86 & 28.65 & 28.84 & 3.99 & 3.96 & 4.26 \\
\hline 135 & 37500 & 28.90 & 28.90 & 28.77 & 28.87 & 3.96 & 3.92 & 4.17 \\
\hline 136 & 27364 & 28.91 & 28.91 & 28.83 & 28.90 & 4.08 & 4.13 & 4.20 \\
\hline 137 & 20266 & 29.01 & 29.01 & 28.90 & 28.94 & 4.05 & 4.10 & 4.17 \\
\hline 138 & 15198 & 28.97 & 28.97 & 28.93 & 28.97 & 4.14 & 4.32 & 4.21 \\
\hline 139 & 11535 & 28.97 & 28.97 & 28.99 & 29.01 & 4.23 & 4.46 & 4.21 \\
\hline 140 & 8866 & 29.02 & 29.02 & 29.02 & 29.05 & 4.21 & 4.51 & 4.24 \\
\hline 141 & 6885 & 29.08 & 29.08 & 29.08 & 29.08 & 4.18 & 4.41 & 4.20 \\
\hline 142 & 5403 & 29.14 & 29.14 & 29.13 & 29.12 & 4.10 & 4.39 & 4.21 \\
\hline 143 & 4283 & 29.16 & 29.16 & 29.17 & 29.17 & 4.19 & 4.68 & 4.30 \\
\hline 144 & 3425 & 29.22 & 29.22 & 29.23 & 29.23 & 4.18 & 4.69 & 4.45 \\
\hline 145 & 2765 & 29.28 & 29.28 & 29.28 & 29.25 & 4.09 & 4.57 & 4.56 \\
\hline 146 & 2250 & 29.35 & 29.35 & 29.34 & 29.31 & 4.11 & 4.52 & 5.10 \\
\hline 147 & 1845 & 29.39 & 29.39 & 29.39 & 29.35 & 4.16 & 4.72 & 5.49 \\
\hline 148 & 1525 & 29.47 & 29.47 & 29.45 & 29.42 & 4.12 & 4.68 & 5.92 \\
\hline 149 & 1268 & 29.56 & 29.56 & 29.53 & 29.46 & 4.18 & 4.58 & 6.53 \\
\hline 150 & 1062 & 29.62 & 29.62 & 29.59 & 29.50 & 4.29 & 4.53 & 7.08 \\
\hline
\end{tabular}


in comparison to the time range that is dominated by a Smoluchowski time dependence. The quality with which the initial part of $I_{\mathrm{DF}}(t)$ is described in a fit of eqn. (12) affects the accuracy with which $R_{\mathrm{ST}}$ can be determined. Therefore the measured curve was divided into nine time sections, which increased monotonically in size and were weighted differently in the fit. The size of the first section was chosen according to the time where $I_{\mathrm{DF}}(t)$ reached approximately half of the maximum intensity value, the size of each following section (with increasing time) was iteratively doubled and adapted to the overall duration of the measurement by a scaling factor. The weighting factors of the nine sections were chosen such that the number of data-points per section multiplied by the weighting factor of the section was constant. Hence the relative sizes of the sections determine their weighting, and the emphasis is thus put on the initial data-points, for the size of sections increases monotonically. The importance of the weighting procedure for balancing the impact of the time ranges of antiSmoluchowski and Smoluchowski temporal behaviour on the data statistics will become apparent in the result section, where the information, which can be extracted from the time dependence of the delayed fluorescence in form of kinetic parameters, will be discussed.

Owing to this improvement the values of the parameters $R_{\mathrm{ST}}$ and $R_{\mathrm{A}}$ do not any longer depend systematically on the time interval in which $I_{\mathrm{DF}}(t)$ is evaluated as opposed to the procedure in 3 (details will be given in the next subsection - see also Fig. 9).

(d) In ref. 3 the first-order triplet-decay rate constant $k_{\mathrm{T}}$ was obtained from the decay of the phosphorescence at $670 \mathrm{~nm}$ and subsequently used as a fixed parameter in the analysis of $I_{\mathrm{DF}}$. In the present work also $k_{\mathrm{T}}$ is a variable parameter and follows from the analysis of $I_{\mathrm{DF}} ; k_{\mathrm{T}}$ will be compared to values of the triplet decay rate constant obtained from the monoexponential decay of the phosphorescence. It serves as a measure for the validity of the modified Smoluchowski model. Fig. 5 shows the phosphorescence, $I_{\mathrm{PH}(\text { hom })}(t)$ and $I_{\mathrm{PH}(\text { per) }}(t)$, of anthracene in $c t-\mathrm{DMCH}$ at $136 \mathrm{~K}$, which were measured simultaneously to $I_{\mathrm{DF}(\mathrm{hom})}(t)$ and $I_{\mathrm{DF}(\mathrm{per})}(t)$, respectively. A single exponential was fitted to the phosphorescence data in order to calculate $I_{\mathrm{PH}, \mathrm{cal}}$, for the respective decays, shown in the upper part of Fig. 5. The lower graphs of Fig. 5 show the weighted residuals, $\Delta_{\mathrm{PH}(k)}=\left(I_{\mathrm{PH}(k)}-I_{\mathrm{PH}(\mathrm{cal})}\right) / I_{\mathrm{PH}(k)}(k=$ hom, per $)$ of the fits to demonstrate the quality of the least square minimization.

\section{The kinetic parameters and their temperature dependence}

For all temperature dependent fits the start values of the variable fit parameters were $R_{\mathrm{A}}=1.0 \mathrm{~nm}, R_{\mathrm{ST}}=4.0 \mathrm{~nm}, k_{\mathrm{T}}=$ $29.0 \mathrm{~s}^{-1}$, and $D=2 D_{\mathrm{SE}}$ based on the results of $D_{0}$ from the spatially periodic excitation at $136 \mathrm{~K}$. The start value for the parameter $b$ was set to 0.2 in the case of periodic excitation. Fixed parameters were $p=0.9995, R_{\mathrm{SS}}=5.0 \mathrm{~nm}$ (practically irrelevant), and $d=\lambda(2 n(t))^{-1} \sim 119 \mathrm{~nm}$ for spatially periodic excitation. The time dependence of the delayed fluorescence and phosphorescence of anthracene in $c t$-DMCH was studied between 134 and $150 \mathrm{~K}$ in steps of $1 \mathrm{~K}$. Over this temperature range the viscosity $\eta$ of the solvent changes by two orders of magnitude $^{12}$ (see Table 1). The absolute values of the kinetic parameters obtained, listed in Table 1 and 2, will be discussed in turn:

The relative diffusion coefficient, $D$. The role of the relative diffusion coefficient $D$ as a function of temperature is a key for the discussion of the applicability and validity of Smoluchowski's model to diffusion controlled TTA. The following procedures to determine $D(t)$ were used and the results are shown in Fig. 6: At first values of $D$ were determined upon spatially periodic excitation at 136 and $142 \mathrm{~K}$ from which an

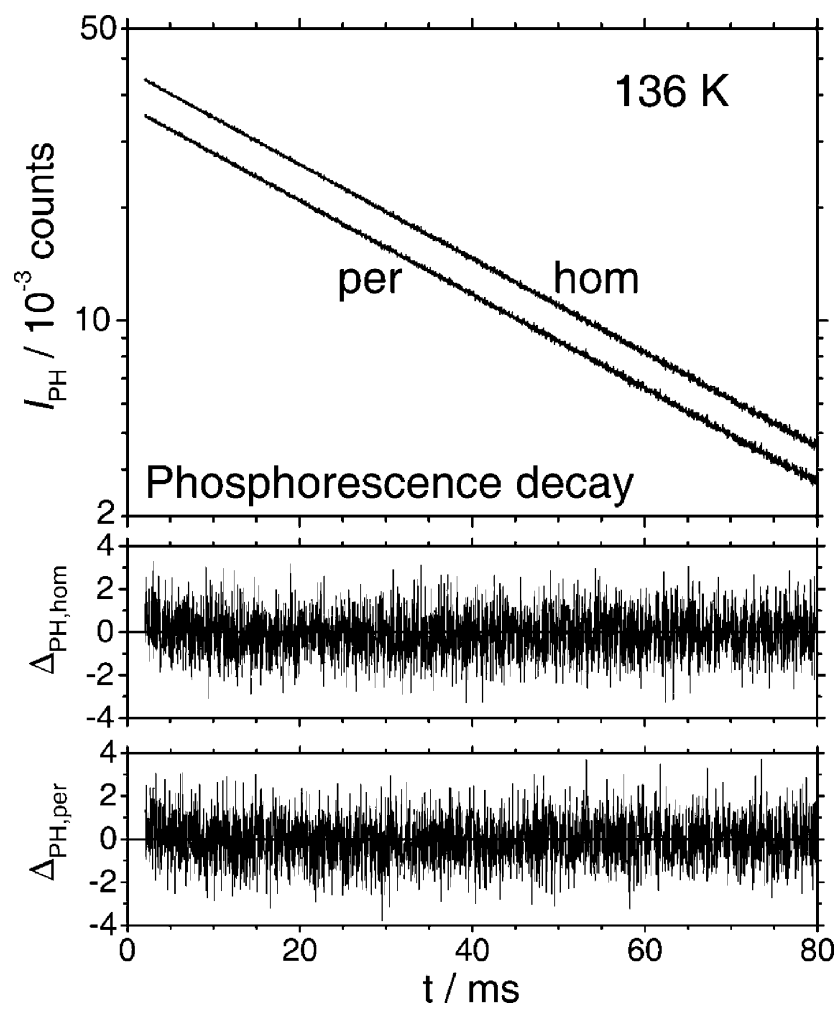

Fig. 5 Phosphorescence $(\mathrm{PH})$ decay of anthracene in $c t$-DMCH at $136 \mathrm{~K}$ upon spatially periodic (per) and homogeneous (hom) excitation. PH simultaneously measured with DF in Fig. 4. Upper graph: traces of measured intensities, $I_{\mathrm{PH}(\mathrm{hom})}$ and $I_{\mathrm{PH}(\mathrm{per})}$, as well as calculated values $I_{\mathrm{PH}, \text { cal }}=A_{\mathrm{PH}} \exp \left(-k_{\mathrm{T}, \mathrm{PH}} t\right)$ for both decays. Fit parameters hom: $A_{\mathrm{PH}}=46510 \pm 15$ counts and $k_{\mathrm{T}, \mathrm{PH}}=28.895 \pm 0.007$ $\mathrm{s}^{-1}$; per: $A_{\mathrm{PH}}=37303 \pm 13$ counts and $k_{\mathrm{T}, \mathrm{PH}}=28.875 \pm 0.008 \mathrm{~s}^{-1}$; Difference in $k_{\mathrm{T}}$ equals $0.07 \%$. Lower graphs: weighted residuals $\Delta_{\mathrm{PH}}=\left(I_{\mathrm{PH}}-I_{\mathrm{PHF}, \mathrm{cal}}\right) / I_{\mathrm{PH}}$ for fits of measurements hom and per. The interrupts created by chopper 3 have been eliminated from the plot.

'effective' radius $r(=0.16 \mathrm{~nm})$ was derived assuming the Stokes-Einstein (SE) equation,

$$
D / 2=D_{\mathrm{SE}}=k_{\mathrm{B}} T(6 \pi \eta r)^{-1},
$$

for spherical particles of radius $r$ to be valid in this temperature regime ( $k$ is the Boltzmann constant). With $r$, the temperature dependence of the (absolute) diffusion coefficient $D_{\mathrm{SE}}$ was calculated with eqn. (17) (open circles ' $O$ ' and dashed line in Fig. 6 , column 4 in Table 1 ). The calculated values $2 D_{\mathrm{SE}}$ served as start parameters for $D$ in the evaluation of curves obtained upon spatially homogeneous excitation. This approach was taken due to the very strong correlation of $D$ and $R_{\mathrm{A}}$. It is expected that the calculated start parameters, $2 D_{\mathrm{SE}}$, are very close to the 'true' diffusion coefficients $2 D_{0}(t)$, which minimize the sum of the squared deviations $\left(\chi^{2}\right)$ in the simplex variation. $\ddagger \ddagger$ In other words, the $D \leftrightarrow R_{\mathrm{A}}$ correlation becomes insignificant by starting the fit as close as possible to the true $\chi^{2}$ minimum. $D_{0}$ of anthracene in $\mathrm{T}_{1}$ in $c t$-DMCH between 134 and $150 \mathrm{~K}$, derived from curves measured upon spatially homogeneous excitation, follows an Arrhenius-like temperature dependence and can be empirically represented by

$$
\begin{aligned}
\log _{10}\left(D_{0} / \mathrm{nm}^{2} \mathrm{~s}^{-1}\right)= & (19.39594 \pm 0.17133) \\
& -(2045.72319 \pm 24.28507 \mathrm{~K}) / T
\end{aligned}
$$

It Note that the corresponding quantities $D_{\mathrm{SE}}$ (calculated) and $D_{0}$ (from fit) are absolute diffusion coefficients of triplet molecules, whereas $D\left(\equiv 2 D_{0}\right)$ in Smoluchowski's equation is the relative diffusion coefficient of two molecules in the triplet state, which is varied in the fit. 


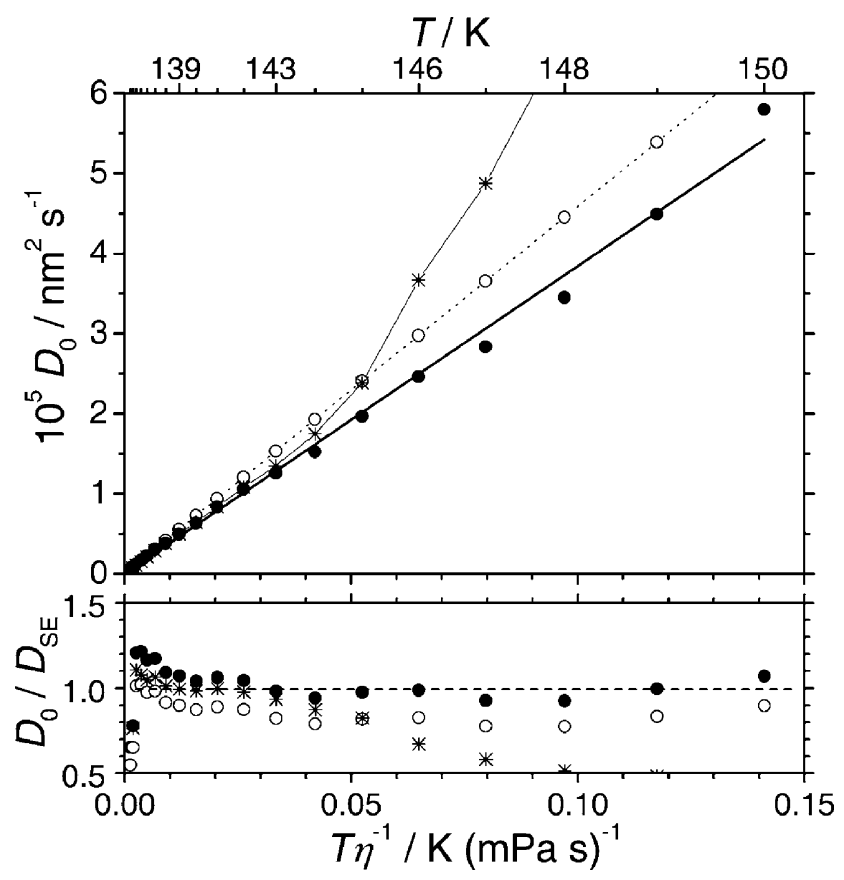

Fig. 6 Plot of $D_{0}(=D / 2) v s$. $T / \eta$. The open circles (O) represent absolute diffusion coefficients, $D_{\mathrm{SE}}$, calculated with the StokesEinstein (SE) equation using an effective particle radius $r_{\text {eff }}=(1.60 \pm$ $0.03) \AA$, which was derived from a measurement of $I_{\mathrm{DF}(\mathrm{per})}$ at $136 \mathrm{~K}$ (Fig. 3). These values were used as start parameters in fits of eqn. (12) to $I_{\mathrm{DF} \text { (hom) }}(t)$, from which the values of $D_{0}$ (solid circles, $\bullet$ ) are derived. An effective particle radius $r_{\text {eff }}=(1.91 \pm 0.03) \AA$ in the Stokes-Einstein relationship was determined from the slope of the straight solid line $(-)$. The stars $(*)$ represent $D_{0}$ values obtained in fits without weighting procedure. The lower viewgraph shows the ratios of diffusion coefficients in the upper graph: Solid circles $=(\bullet /$ solid line $)$ open circles $=(\bullet / \bigcirc)$, stars $=(\bullet / *)$. The (interpolated) viscosity values $\eta$ were taken from ref. 12 .

with a standard deviation of the fit of 0.0244 (column 1 in Table 1). Values of the absolute diffusion coefficient are shown in Fig. 6 (solid circles ' $\bullet$ '). The solid line in Fig. 6 shows a fit of eqn. (17) to $D_{0}$ as a function of $T / \eta$ (solid line) yielding an effective particle radius of $r=1.9 \pm 0.1 \AA$, which is quite close to a value found for triplet anthracene in hexane at higher temperatures. ${ }^{9}$ For non-spherical solute molecules eqn. (17) should in principle reproduce the measured values of $D_{0}$ within a factor of $2^{ \pm 1}$, if the sizes of solvent and solute molecules are similar and an appropriate value for $r$ is applied. This criterion holds for the temperature range in this study, although deviations from the Stokes-Einstein law become apparent at low temperatures. It is known that significant deviations from the Stokes-Einstein equation, which cannot be determined a priori, exist for aromatic molecules. ${ }^{19}$ The bottom panel of Fig. 6 shows the ratios $D_{0} / D_{\mathrm{SE}}$, where the solid circles demonstrate the deviation of the description of $D_{0}$ with respect to the Stokes-Einstein equation. The open circles illustrate the change from the start parameters (derived from the measurements upon spatially periodic excitation) in the evaluation procedure, which is between a factor $\approx 0.8$ and $\approx 1.0$ for all but the lowest two temperatures. Below $138 \mathrm{~K}$ the ratio $D_{0} / D_{\mathrm{SE}}$ remains significantly larger than unity and finally drops at the lowest measured temperatures. Since the approximation of anthracene as a spherical molecule seems to be justified at higher temperatures, the most likely explanation for this deviation at low temperatures is the invalidity of the StokesEinstein law in this temperature region where structural changes of the solvent in the vicinity of the glass transition start to play a role. Due to the increase in viscosity of $c t$ $\mathrm{DMCH}$ by a factor of $\approx 100$ over the studied temperature range the diffusion properties of the system are likely to be affected. (The accuracy of the viscosity data is better than $\pm 2 \%$ in this temperature region ${ }^{12}$ ). In a critical temperature range, whose onset may well be covered by our measurements, translational diffusion may be sustained by areas of free volume forming in the cooling (contracting) process of the liquid $^{20,21}$ and thus leading to an increase in $D_{0} / D_{\mathrm{SE}}$. A perfect agreement with the Stokes-Einstein equation over the entire temperature region can therefore not be expected. Due to the remaining uncertainty of values of the diffusion coefficient, the temperature dependence $D(T)$ alone is in fact inappropriate to corroborate the validity regime of Smoluchowski's theory in the context of our experiments concerning the kinetics of TTA.

The importance of weighting data in the fit procedure for the value of $D_{0}$ is also demonstrated in Fig. 6: The stars in the upper graph correspond to values of $D_{0}$ obtained without weighting in the fit (column 5 in Table 1), and in the lower graph to the ratio $D_{0}$ (with weighting) $/ D_{0}$ (without weighting). It is obvious that the weighting is certainly essential to fully account for the initial condition eqn. (7) at higher temperatures, where the fits without appropriate weighting procedure become much worse resulting in a very substantial deviation of $D(T)$ from a Stokes-Einstein behaviour. At lower temperatures, where the anti-Smoluchowski behaviour takes up a considerable part of $I_{\mathrm{DF}(\mathrm{hom})}(t)$, fits without weighting the data seem to yield satisfactory results.

(The use of symbols in the remaining text will be according to the different evaluation procedures of $I_{\mathrm{DF}}(t)$ outlined in this section. Open circle ' $O$ ': absolute diffusion coefficient $D_{\mathrm{SE}}$ of anthracene in $T_{1}$ calculated with $r=0.16 \mathrm{~nm}$ in eqn. (17) as derived from measurements upon spatially periodic excitation ( $D_{\mathrm{SE}}$ is fixed in the fit). Solid circle ' $\bullet$ ': Value of $D_{\mathrm{SE}}$ is used as start parameter, however all parameters are varied and the weighting procedure is applied. Star ' $*$ ': Value of $D_{\mathrm{SE}}$ is used as start parameter, however all parameters are varied but no weighting procedure is applied.).

The rate coefficient of the triplet decay, $\boldsymbol{k}_{\mathrm{T}}$. Since the time dependence of the delayed fluorescence of anthracene in ct$\mathrm{DMCH}$ was measured simultaneously to the phosphorescence decay, the comparison of the first-order triplet decay rate coefficient $k_{\mathrm{T}}$ as determined independently from both measurements is a very convenient way to evaluate the temperature regime in which Smoluchowski's modified theory is well applicable to TTA. Fig. 7 shows $k_{\mathrm{T}, \mathrm{DF}}\left(=k_{\mathrm{T}}\right.$ as determined from the delayed fluorescence) and $k_{\mathrm{T}, \mathrm{PH}}\left(=k_{\mathrm{T}}\right.$ derived from the monoexponential phosphorescence decay) as a function of temperature. The agreement between the values of $k_{\mathrm{T}, \mathrm{DF}}$ and $k_{\mathrm{T}, \mathrm{PH}}$ is so good that the average deviation over the entire temperature range is smaller than $\pm 0.17 \%$. For half of the temperatures the correspondence is better than $0.1 \%$ (lower graph in Fig. 7). This quite impressive accuracy shows the applicability of Smoluchowski's theory over most of the temperature range of interest. Only at the lowest temperatures the viscosity is so high that the assumption of TTA being diffusion controlled may no longer be valid. Of all relevant parameters the triplet decay is the most independent one in terms of parameter correlation. For instance, fixing the diffusion coefficient at its start value $2 D_{\mathrm{SE}}$ (Fig. 6) in the fit procedure, as opposed to varying it, results in a change of $k_{\mathrm{T}}$ of maximal $\pm 0.04 \%$ at all temperatures, as long as a weighting procedure is applied. The stars in Fig. 7 again represent values of $k_{\text {T,DF }}$ that were obtained without applying the weighting procedure in the fit. The deviation from $k_{\mathrm{T}, \mathrm{PH}}$ increases as the time dominated by anti-Smoluchowski behaviour at low temperatures increases due to slowing diffusion, i.e. less data-points are available from which information on $k_{\mathrm{T}}$ can be extracted. In ref. $3 k_{\mathrm{T}}$ was not even treated as a variable fit-parameter. A summary of values found for $k_{\mathrm{T}}$ are listed in columns 3-6 in Table 2 . 


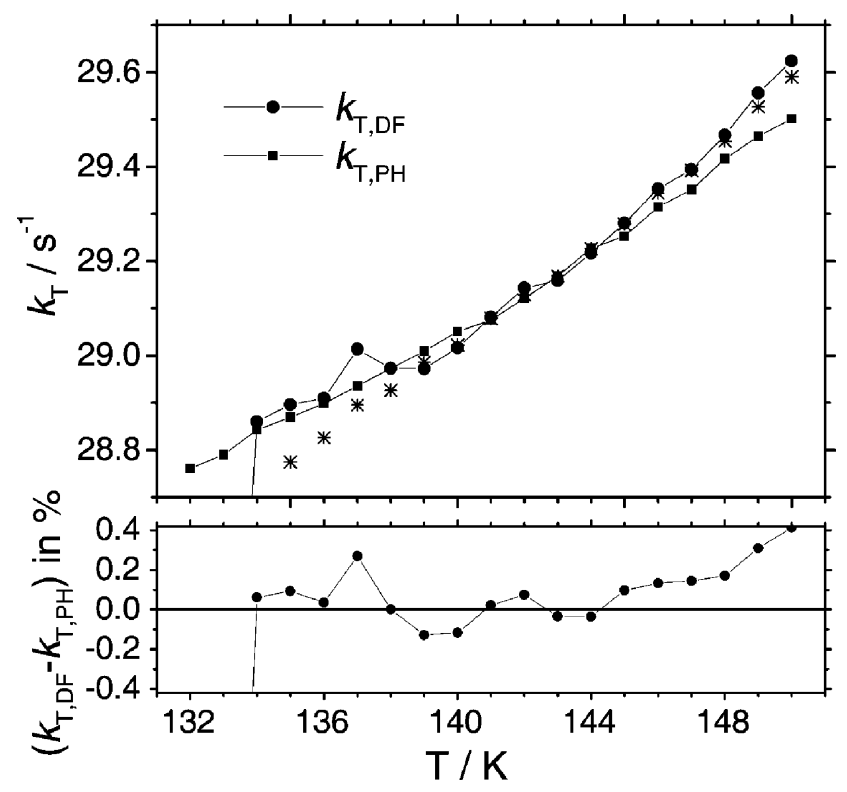

Fig. 7 Temperature dependence of the triplet decay rate constant $k_{\mathrm{T}}$ as obtained from the fit of eqn. (12) to the time dependence of the delayed fluorescence ( $k_{\mathrm{T}, \mathrm{DF}}$, solid circles, hom) in comparison with values obtained directly from the phosphorescence decay $\left(k_{\mathrm{T}, \mathrm{PH}}\right.$, solid squares). The stars represent values of $k_{\mathrm{T}, \mathrm{DF}}$ that were obtained without applying the weighting procedure in the evaluation of $I_{\mathrm{DF}}(t)$. The lower graph represents the difference between $k_{\mathrm{T}, \mathrm{DF}}(\bullet)$ and $k_{\mathrm{T}, \mathrm{PH}}(\boldsymbol{\square})$ in percent.

The Förster and the effective annihilation radii, $\boldsymbol{R}_{\mathrm{ST}}$ and $\boldsymbol{R}_{\mathrm{A}}$. The values of the parameters $R_{\mathrm{ST}}$ and $R_{\mathrm{A}}$ do not (any longer) depend systematically on the time interval $f_{\mathrm{i}}=\left(t_{1}, t_{2}\right)$ in which $I_{\mathrm{DF}}(t)$ is evaluated as opposed to the procedure in ref. 3. Evidence for this improvement is given in Fig. 8, where $I_{\mathrm{DF}}(t)$ at $141 \mathrm{~K}$ was analysed in five different time intervals $f_{1}=(0.05$, $81 \mathrm{~ms}), f_{2}=(0.05,56 \mathrm{~ms}), f_{3}=(0.05,27 \mathrm{~ms}), f_{4}=(0.05,9.9$ $\mathrm{ms})$ and $f_{5}=(0.05,3.1 \mathrm{~ms})$, the results are summarized in Table 3 . There are two reasons why the fit parameters $R_{\mathrm{ST}}$ and $R_{\mathrm{A}}$ do not depend critically on the time range in which the delayed fluorescence is evaluated: (i) The binary approximation for the TTA is valid under the present experimental conditions as opposed to ref. 3 , where a breakdown of the binary approximation at high temperatures could not be ruled out. This is not surprising since the maximum initial triplet concentration $\left[\mathrm{T}_{1}\right]_{0}$ was estimated in the experimental section to be $\approx 100$ times lower than in ref. 3 , and hence for all temperatures the validity condition $k_{\mathrm{T}} \gg k_{2 \mathrm{~A}}(\infty)\left[\mathrm{T}_{1}\right]_{0}$ for eqn. (12) holds (even at $150 \mathrm{~K}$ the ratio $\left.k_{\mathrm{T}} /\left(k_{2 \mathrm{~A}}(\infty)\left[\mathrm{T}_{1}\right]_{0}\right) \approx 700\right)$. Additionally the anthracene concentration was somewhat lower than in ref. 3 such that saturation effects are certainly negligible. (ii) The implementation of the weighting procedure also essentially improved the data evaluation. Small changes in the time dependence of $k_{2 \mathrm{~A}}(t)$ can cause significant changes in the parameter values. The behaviour of $k_{2 \mathrm{~A}}(t)$ normalized to $k_{2 \mathrm{~A}}(\infty)$ is shown in the insert in Fig. 8 for the first millisecond only. On one hand the entire information on $R_{\mathrm{A}}$ is contained in the small deviation of $k_{2 \mathrm{~A}}(t)$ from $k_{2 \mathrm{~A}}(\infty)$ in the time regime of Smoluchowski behaviour. At all temperatures more than $97 \%$ of the data-points of $I_{\mathrm{DF}}(t)$ are in that time regime (not shown in the insert in Fig. 8). It should therefore be possible to use the temperature dependence of $R_{\mathrm{A}}$ as another indicator for the validity regime of the modified Smoluchowski approach. On the other hand $R_{\mathrm{ST}}$ can only be evaluated accurately, if the entire anti-Smoluchowski time regime is taken into account. The new weighting procedure balances this difficulty, certainly at medium to high temperatures in the present study. In order to appreciate the quality enhancement of the fit procedure, the residuals and ratios $f_{1} / f_{i}(i=2-5)$ in Fig. 8 ought to be compared with Fig. 11 in ref. 3.

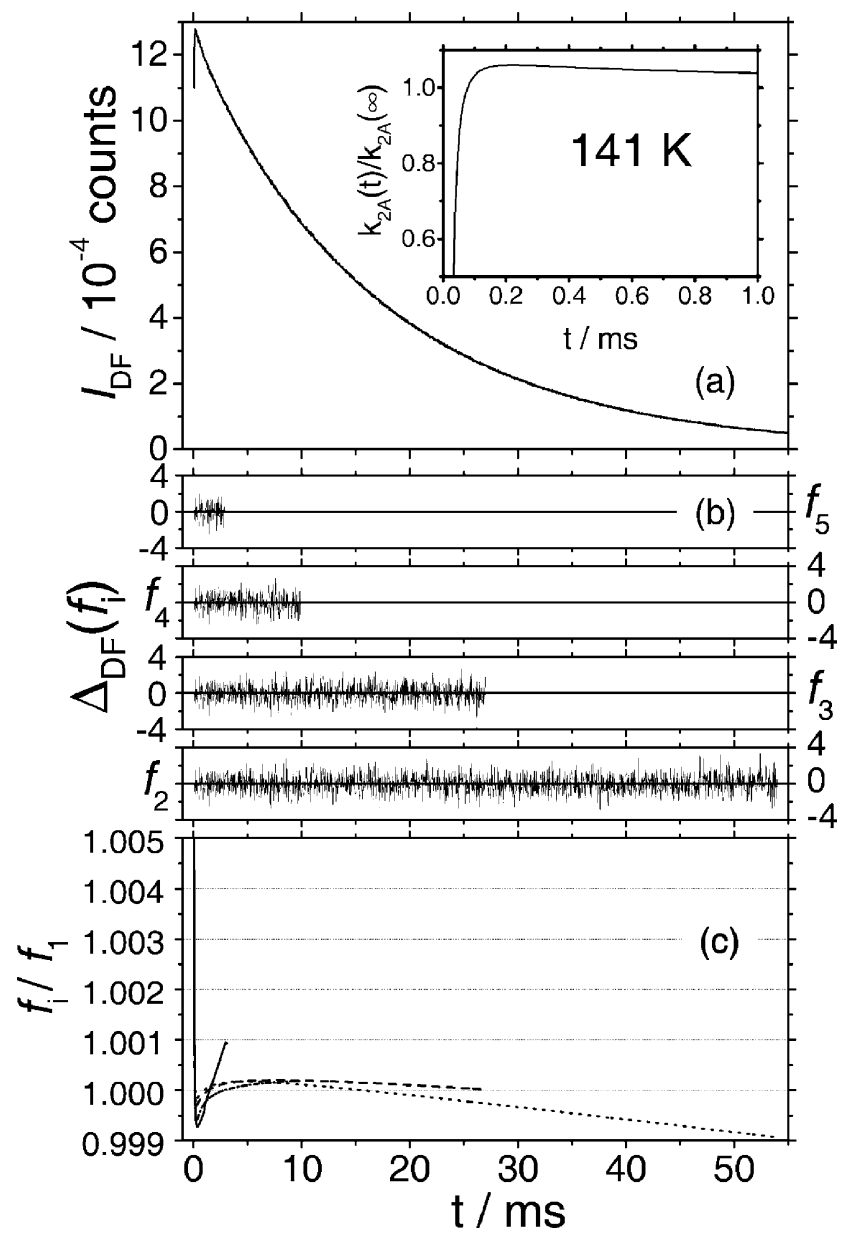

Fig. 8 (a) Time dependence of the intensity $I_{\mathrm{DF}(\mathrm{hom})}$ of the delayed fluorescence from anthracene in $c t$-DMCH at $141 \mathrm{~K}$. Calculated curves are actually included in the graph according to fits in different time intervals $f_{i}=(0.05,56 \mathrm{~ms})(0.05,27 \mathrm{~ms})(0.05,9.9 \mathrm{~ms})$ and $(0.05,3.1$ $\mathrm{ms})$, parameter values are listed in Table 3; (b) residuals $\Delta_{\mathrm{DF}}=\left(I_{\mathrm{DF}}-I_{\mathrm{DF}, \text { al }}\left(f_{\mathrm{i}}\right)\right) / \frac{1}{I_{\mathrm{DF}}^{2}}$ for ranges $f_{2}-f_{5}$ (the full range fit, $f_{1}$, as shown in Fig. 4 for $T=136 \mathrm{~K}$, is not displayed) (c) The calculated curves $f_{2}-f_{5}$ are represented as ratios $f_{\mathrm{i}} / f_{1}$. Compare the quality of the fits and the randomness of the deviations with Fig. 11 and Table 2 in ref. 3 respectively.

Based on Smoluchowski's original model, $D$ is the only parameter that necessarily depends on the temperature. The steady state value of the average distance at which TTA takes place, $R_{\mathrm{A}}(\infty)$, is in first approximation expected to be independent of the temperature as well as the Förster radius $R_{\mathrm{ST}}$. However, as already experimentally indicated in refs. 3 and $4, R_{\mathrm{A}}$ and $R_{\mathrm{ST}}$ slightly decrease with increasing temperature. This result is experimentally corroborated in this study. Both radii as obtained from evaluations of $I_{\mathrm{DF}(\text { hom })}(t)$ are shown as a function of temperature in Fig. 9. The choice of all symbols in Fig. 9 is in line with that for Fig. 6, both figures are

Table 3 Dependence of the parameters $R_{\mathrm{ST}}$ and $R_{\mathrm{A}}$ at $141 \mathrm{~K}$ on the time interval $\left(t_{1}, t_{2}\right)$ used for curve-fitting (see Fig. 8). All parameters ( $R_{\mathrm{ST}}, R_{\mathrm{A}}, D$ and $k_{\mathrm{T}}$ ) were treated as variable parameters in the curve-fitting; $\sigma$ is the standard deviation of a fit

\begin{tabular}{lrrlllll}
\hline & $t_{1} / \mathrm{ms}$ & $t_{2} / \mathrm{ms}$ & $R_{\mathrm{ST}} / \mathrm{nm}$ & $R_{\mathrm{A}} / \mathrm{nm}$ & $D / \mathrm{nm}^{2} \mathrm{~s}^{-1}$ & $k_{\mathrm{T}} / \mathrm{s}^{-1}$ & $\sigma$ \\
\hline$f_{1}(t)$ & 0.056 & 81.91 & 4.18 & 0.99 & 166941 & 29.08 & 1.9800 \\
$f_{2}(t)$ & 0.056 & 53.97 & 4.17 & 0.97 & 167840 & 29.09 & 1.9726 \\
$f_{3}(t)$ & 0.056 & 26.97 & 4.16 & 0.97 & 167623 & 29.09 & 1.9965 \\
$f_{4}(t)$ & 0.056 & 9.89 & 4.11 & 0.96 & 164136 & 29.08 & 1.9458 \\
$f_{5}(t)$ & 0.056 & 3.08 & 4.21 & 0.99 & 170389 & 28.78 & 1.8757 \\
\hline
\end{tabular}




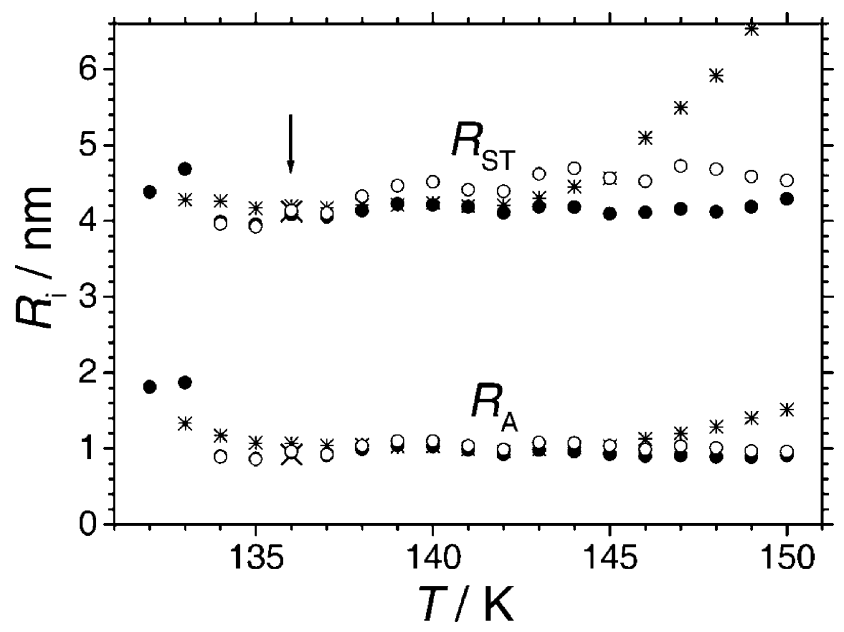

Fig. 9 Upper data-points: $R_{\mathrm{ST}} v s$. temperature. Lower data-points: $R_{\mathrm{A}} v s$. temperature. The values of $R_{i}(i=\mathrm{A}, \mathrm{ST})$ are derived from fits of eqn. (12) to $I_{\mathrm{DF}(\mathrm{hom})}(t)$ with start parameters $D=2 D_{\mathrm{SE}}$ (open circles in Fig. 6). The solid and open circles correspond to results where $D$ was varied and fixed at $2 D_{\mathrm{SE}}$ in the fully weighted fit, respectively. The values corresponding to the star symbols were obtained with $D$ varied but no weighting of the fit. The vertical arrow (symbol X) indicates the values obtained at $136 \mathrm{~K}$ from $I_{\mathrm{DF}(\mathrm{per})}(t)$ (all parameters were varied and the fit weighted)

based on the same fits. Even though $D$ was fixed at StokesEinstein values $2 D_{\mathrm{SE}}$ (column 4 in Table 1) in one set of evaluations of $I_{\mathrm{DF}(\mathrm{hom})}(t)$ (open circles), and varied in the other (solid circles), the corresponding values for $R_{\mathrm{A}}$ deviate by not more than $10 \%$ (column 7 and 8 in Table 1), although the quality $\left(\chi^{2}\right)$ of the fit remained virtually the same. Despite the fact that the correlation of $R_{\mathrm{A}}$ and $D$ is not eliminated in fits of $I_{\mathrm{DF}(\mathrm{hom})}(t)$, the minimum $\chi^{2}$ yields values for $D$ and $R_{\mathrm{A}}$ that are quite close to the start parameters of the fit since the latter are based on the independent measurements with periodic excitation of $I_{\mathrm{DF}}$. The temperature dependence of the radii and their absolute values are in line with expectation. The effective annihilation radius varies between $R_{\mathrm{A}} \approx 1.05$ and $0.88 \mathrm{~nm}$ and gradually approaches the approximate encounter distance $(R \approx 0.7 \mathrm{~nm})$ at the highest temperatures measured. The values of $R_{\mathrm{A}}$ are in agreement with the range in which the Dexter energy transfer mechanism ${ }^{22}$ (based on exchange interaction) operates efficiently, since it requires a sufficient overlap of the orbitals of the triplet state molecules for the spin exchange. Since the Onsager radius, $a$, for the type of molecule such as anthracene is of the order of $0.4-0.5 \mathrm{~nm},{ }^{23}$ the annihilation radius $R_{\mathrm{A}}$ is expected to be approximately of the size of the Onsager cavity, hence $2 a$, which is in agreement with our results. The values obtained for $R_{\mathrm{ST}}$ are similar but slightly smaller than the ones observed in ref. 3; they vary between $\sim 4.2$ and $\sim 4.0 \mathrm{~nm}$, with exception at the lowest temperatures. The radii $R_{\mathrm{ST}} \approx 4.0 \mathrm{~nm}$ agree quite well with a value of $3.9 \mathrm{~nm}$, calculated with Förster's equation (eqn. (13b) in ref. 4) using known spectroscopic data (see Fig. 4 in ref. 4) for anthracene in an alkane solvent. This agreement is relevant since for nonpolar solvents the Förster radius is not expected to depend strongly on the solvent.

Due to the change of the diffusion behaviour owing to the strong change in $\eta$ at the extreme temperatures in this study, the time dependence of $k_{2 \mathrm{~A}}$ as described by Smoluchowski's theory may be significantly affected at the highest and lowest temperatures investigated. The temperature range in which the current approach is valid is hence restricted to 149 $\mathrm{K}<T<135 \mathrm{~K}$. This becomes evident by the substantial deviations of the radii below $135 \mathrm{~K}$, i.e. at the highest viscosities. For two measurements at $T=133$ and $132 \mathrm{~K}$ the kinetic parameters were not even physically meaningful any longer - these measurements are not discussed in this paper. The restricted validity regime at low temperatures could be caused by (i) a genuine effect due to structural changes of the solvent, or (ii) it may be due to the formation of microcrystals, which finally may start to play a role if the sample is cooled to low temperatures for very long periods of time.

The star symbols in Fig. 9 denote values of radii which were obtained when all parameters are varied however without applying the weighting procedure. Values of $\chi^{2}$ obtained with this type of evaluation are significantly worse at temperatures $>145 \mathrm{~K}$, and particularly the Förster radii $R_{\mathrm{ST}}$ increase in an unphysical manner with increasing temperature. At low temperatures, where the number of $I_{\mathrm{DF}}$ data-points belonging to Smoluchowski and anti-Smoluchowski behaviour are more balanced, the parameters have physically reasonable values and hence fits without weighting procedure become acceptable if not superior (see also below).

\section{TTA in terms of the model by Butler and Pilling ${ }^{10}$}

In the previous section it was shown, that the time dependence of the delayed fluorescence can be satisfactorily described by the kinetic model based on Smoluchowski's modified kinetic theory, particularly if data for the determination of $D$ through independent measurements (such as spatially periodic excitation of $I_{\mathrm{DF}}$ ) are available. This modified Smoluchowski model can essentially be compared with an approach by Butler and Pilling: ${ }^{10,24}$ In their model the energy transfer process of TTA is assumed to be based on exchange interaction, ${ }^{22}$ with a distance-dependent first order rate coefficient

$$
k_{\mathrm{A}}(r)=A \exp (-2 r / L),
$$

where $A$ (frequency factor) and $L$ (characteristic length) are intrinsic empirical parameters of the system. Consequently Fick's second law and Smoluchowski's boundary condition eqn. (5) are replaced by ${ }^{24}$

$$
\begin{gathered}
\frac{\partial \rho_{\mathrm{A}}(r, t)}{\partial t}=\frac{D}{r} \frac{\partial^{2}\left[r \rho_{\mathrm{A}}(r, t)\right]}{\partial r^{2}}-A \mathrm{e}^{-2 r / L} \rho_{\mathrm{A}}(r, t), \\
\text { with }\left.\quad \frac{\partial \rho_{\mathrm{A}}(r, t)}{\partial r}\right|_{r=R}=0 .
\end{gathered}
$$

The new boundary condition eqn. (21), which implies that the relative diffusion of triplet state molecules is restricted to distances $r$ larger or equal to the encounter distance $R$, is well justified in viscous solution, since TTA occurs at distances $r>R$. In ref. 3 eqn. (20) was solved numerically using the initial condition (7) and boundary condition (21), and expressions were derived for the normalized radial TTA rate density, $v_{\mathrm{A}}(r, t)$, the time-dependence of the second-order rate coefficient, $k_{2 \mathrm{AP}}^{\mathrm{BP}}(t)$, and the mean time-dependent intermolecular distance, $R_{\mathrm{A}}^{\mathrm{BP}}(t)$, for TTA (the superscript BP stands for the approach by Butler and Pilling). The steady-state limit of $R_{\mathrm{A}}^{\mathrm{BP}}(t)$ can be described by ${ }^{2,24}$

$$
R_{\mathrm{A}}^{\mathrm{BP}}(\infty) \equiv R_{\mathrm{eff}}=L\left[\gamma+\ln \left(A L^{2} / 4 D\right)\right],
$$

if the condition

$$
\left(A L^{2} / D\right)^{1 / 2} \exp (-R / L) \equiv w_{0}>2
$$

is satisfied ( $\gamma$ is Euler's constant $=0.57721 \ldots$ ). Eqn. (22) was used to determine values for the parameters $A$ and $L$ in a plot of $R_{\mathrm{A}} v s . \log _{10} D$ as shown Fig. 10. According to eqn. (22) the radii $R_{\mathrm{A}}$ must decrease with increasing $D$. In Fig. 10 values of $R_{\mathrm{A}}$ and $D$ are shown, which were determined between 150 and $134 \mathrm{~K}$, using three different ways to evaluate $I_{\mathrm{DF}}(t)$ :

(A) The solid circles $(\bullet)$ were obtained by varying $R_{\mathrm{A}}$ and $D$ (using Stokes-Einstein start-parameter values) and the outlined weighting procedure was applied. For temperatures above $\approx 140 \mathrm{~K}, R_{\mathrm{A}}$ decreases as expected in the BP-model for increasing $D$ values. Below $140 \mathrm{~K}$ however, values of $R_{\mathrm{A}}$ 


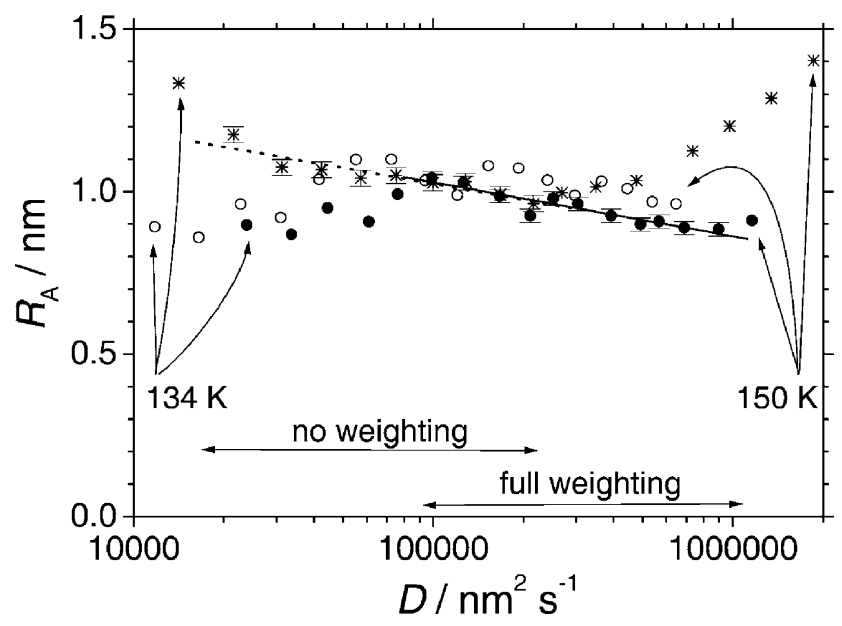

Fig. 10 Plot of $R_{\mathrm{A}}$ vs. $D$ (logarithmic scale). The circles correspond to fit results of $R_{\mathrm{A}}$ and $D$ obtained with full weighting of different time regions, the stars were derived without weighting. For all solid symbols the start parameter for the diffusion coefficient $\left(2 D_{\mathrm{SE}}\right.$ in Fig. 6) were varied in the fit, the open symbols correspond to fixed start parameters according to the Stokes-Einstein relationship. From the straight line fitted to the experimental data using eqn. (22), values of $A=$ $11.641 \times 10^{(12 \pm 0.8)} \mathrm{s}^{-1}$ and $L=(0.142 \pm 0.022) \mathrm{nm}$ are derived for the stars (dotted line, no weighting - fit region indicated); $A=$ $9.576 \times 10^{(12 \pm 0.7)} \mathrm{s}^{-1}$ and $L=(0.144 \pm 0.018) \mathrm{nm}$ are obtained for the solid circles (solid line, full weighting - fit region indicated). The measurements at the extreme temperatures 134 and $150 \mathrm{~K}$ are indicated for the different evaluation procedures. The standard deviation of the fit is 0.0247 . The vertical and horizontal error bars correspond to the uncertainty due to the fit parameters $A$ and $L$, and a $\approx 15 \%$ accuracy of $D$, respectively.

tend to decrease with decreasing $D$ values. The latter, unexpected behaviour according to the BP-model, can have two reasons: (i) the observed deviation is an artefact, because the weighting procedure as outlined above may overrate the time range of anti-Smoluchowski behaviour at low temperatures, hence a different empirical weighting may be required. This explanation only follows from the expected behaviour of $R_{\mathrm{A}}$ according to eqn. (22). Other weighting methods are necessary to clarify this point. (ii) Entirely diffusion controlled conditions are unlikely to be present over the full temperature range studied. Particularly at low temperatures, where deviations from the Stokes-Einstein relationship indicate structural changes in the onset of the glass-transition of the solvent (see Fig. 6), TTA will increasingly be based on long-range energy transfer rather than on exchange interaction (also see ref. 24). Deviations from eqn. (22), which represents the BP-model, may thus be observed in this temperature regime. Photoselection, due to an anisotropic distribution of orientations of triplet molecules immediately after excitation with polarized laser light, does not have an effect on TTA in this investigation. This becomes apparent considering the fact that the relative diffusion length $L_{\mathrm{diff}}=\left(6 \mathrm{D} \tau_{\mathrm{OR}}\right)^{1 / 2}$ is a factor of $\sqrt{3}=1.73$ smaller than the encounter radius $R$ within the orientational relaxation time $\tau_{\mathrm{OR}} \approx \pi R^{3} \eta /\left(12 k_{\mathrm{B}} T\right)$ (Debye-Einstein) of the triplet molecules (if spherical particles are assumed). ${ }^{4}$ Since $R_{\mathrm{ST}}$ and $R_{\mathrm{SS}}$ are 4-5 times larger than $L_{\mathrm{diff}}$, mechanisms other than long-range Förster energy transfer are not conceivable in the anti-Smoluchowski time range. Hence all triplets are randomly oriented at times of dominant Smoluchowski kinetics. The significance of the molecules orientation is discussed in detail in ref. 25.

(B) The open circles (O) correspond to fits where $D=2 D_{\mathrm{SE}}$ was fixed to follow exactly Stokes-Einstein behaviour and only $R_{\mathrm{A}}$ was varied. Qualitatively $R_{\mathrm{A}}(D)$ resulting from these fits (open circles) follows the values of $R_{\mathrm{A}}(D)$ discussed under (A) (solid circles). However, the variations of $R_{\mathrm{A}}(D)$ (open circles) are larger, even in a temperature regime, which yields "well-behaved" results in terms of the BP-model. This difference is a consequence of the variation of $D$ during the fit and is evident by the difference between the solid and the open circles in Fig. 6.

(C) The stars (*) were obtained by varying $R_{\mathrm{A}}$ and $D$ (using Stokes-Einstein start-parameter values) and the weighting procedure was not applied. In this case the values of $R_{\mathrm{A}}$ increase with increasing temperature above $143 \mathrm{~K}$ in an unphysical manner, which agrees with the fact that also no Stokes-Einstein behaviour was obtained for the corresponding $D$ values in this temperature range (see stars in Fig. 6). Hence at high temperatures the weighting procedure is essential for the correct evaluation of $I_{\mathrm{DF}}(t)$. At low temperatures however, the $R_{\mathrm{A}}$ values follow the expected behaviour according to the $\mathrm{BP}$ model. Approximately at the temperature where the fit results obtained with the weighting procedure start to deviate from the form of eqn. (22), the results obtained without the weighting procedures continue to be conform with the $\mathrm{BP}$ model (see (A) above).

The solid and dotted lines in Fig. 10 represent fits of eqn. (22) to the data in "well-behaved" regions (points with error bars) that were obtained with full weighting between 149 and $140 \mathrm{~K}$ (solid circles), and without weighting between 135 and $143 \mathrm{~K}$ (solid squares). Generally eqn. (22) cannot be expected to hold over the entire temperature range, even though its application is in principle justified across the measured temperature range according to eqn. (23), since the factor $w_{0}$ is always larger than 3 (even at $T=150 \mathrm{~K}, w_{0} \approx 3.2$, see Fig. 11 ) assuming a reasonable encounter distance of $R \approx 0.7 \mathrm{~nm}$. There is a temperature regime, where the fit results for $R_{\mathrm{A}}$ and $D$ are insensitive to the nature of the start parameters and weights used in the fit (139-144 K). Extrapolation of eqn. (22) to higher and lower temperatures is however basically impossible: At higher temperatures $R_{\mathrm{A}}$ is naturally limited by the encounter distance $R \approx 0.7 \mathrm{~nm}$. TTA may also not be diffusion-controlled any longer at low viscosities $(T>150 \mathrm{~K})$. Moreover, eqn. (22) may not be applicable any longer in its present form when $w_{0}$ approaches the value of 2 . At lower temperatures, i.e. high viscosities, effects resulting from the increasing rigidity of the matrix can affect the diffusion properties of the system. Deviation due to gradual changes in the annihilation mechanism will also play a role. Hence, for the

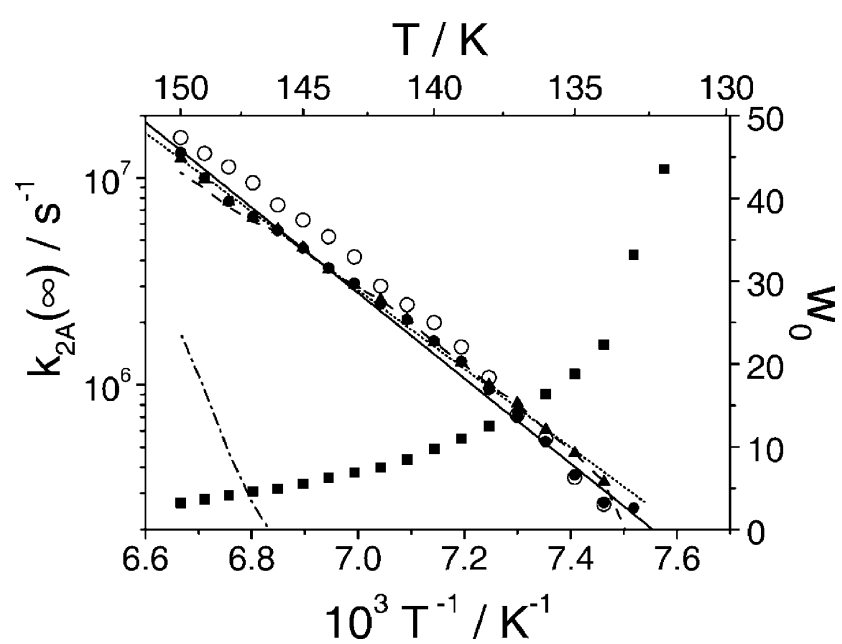

Fig. 11 Left axis: Arrhenius plot of $k_{2 \mathrm{~A}}^{\mathrm{Smol}}(\infty)$ vs. $T^{-1}$. Open and solid circles are due to fit results where the start parameter $D=2 D_{\mathrm{SE}}$ (Fig 3 ) was fixed and varied, respectively. The triangles represent values of $k_{2 \mathrm{~A}}^{\mathrm{BP}}(\infty)$ calculated with eqn. $(24)^{3}$ for $R \approx 0.7 \mathrm{~nm}$. The error of $k_{2 \mathrm{~A}}^{\mathrm{BP}}(\infty)$ for $\Delta R= \pm 0.1 \mathrm{~nm}$ is within the size of the triangular symbols. The main contribution $k_{2 \mathrm{~A}, T}^{\mathrm{BP}}(\infty)$ (dashed line) and $k_{2 \mathrm{~A}, D}^{\mathrm{BP}}(\infty)$ (dashdotted line) are also shown. The straight lines are Arrhenius fits to the triangles (dotted line) and solid circles (solid line) - activation energies are stated in the text. Right axis: Factor $w_{0}>2$ for all temperatures according to eqn. $(23)^{24}$ (solid squares). 
fit of eqn. (22) to the data, points measured at the extreme temperatures (below $135 \mathrm{~K}$ and at $150 \mathrm{~K}$ ) were omitted. Two measurements at $T=133 \mathrm{~K}$ and $132 \mathrm{~K}$ were also not discussed in this paper, because the analysis did not yield meaningful values, particularly for the radii obtained. The values obtained for $A$ and $L$ are $9.576 \times 10^{(12 \pm 0.7)} \mathrm{s}^{-1}$ and $0.144 \pm 0.018 \mathrm{~nm}$ (solid line), as well as $11.641 \times 10^{(12 \pm 0.8)} \mathrm{s}^{-1}$ and $0.142 \pm$ $0.022 \mathrm{~nm}$ (dashed line) respectively - the error limits are due to the fit. Although $A$ and $L$ strongly correlate and their accuracy depends on the uncertainty of the temperature dependence of $R_{\mathrm{A}}$ as obtained from the analysis of experimental data with and without weighting procedure, the values for $A$ and $L$ agree surprisingly well in the chosen temperature regions. The values for $A$ and $L$ are somewhat larger than a range of feasible values found in ref. 3 for anthracene in MCH-MCP: $\quad 2 \times 10^{13} \mathrm{~s}^{-1} \leq A \leq 2 \times 10^{16} \mathrm{~s}^{-1}$ and 0.20 $\mathrm{nm} \geq L \geq 0.15 \mathrm{~nm}$. This small discrepancy can have the following reasons: (i) Although the relative diffusion coefficients of triplet anthracene are comparable in $c t$-DMCH and $\mathrm{MCH}-$ MCP, they will possess a somewhat different temperature dependence due to the different viscosity of the two solvents. (ii) In ref. 3, $D$ values were extrapolated from room temperature and were thus not as accurate as in the present case. (iii) In Fig. 10 it is apparent that the results based on exact Stokes-Einstein behaviour of $D$ (fixed in the fit) were the least accurate one in the present study. In solid solution the frequency factor $A$ seems to be somewhat smaller. For instance in a publication by Inokuti and Hirayama, ${ }^{26}$ who analyzed data by Ermolaev and Terenin on naphthalene in alcoholether at $77 \mathrm{~K}$, values of $L=0.143 \mathrm{~nm}$ and $A \sim 1-6 \times 10^{10}$ $\mathrm{s}^{-1}$ can be found. ${ }^{27,28}$ Personov and coworkers ${ }^{29}$ studied coronene at $77 \mathrm{~K}$ in an organic glass and found values of $A=10^{(8.2 \pm 1.0)} \mathrm{s}^{-1}$ and $L=0.54 \pm 0.10 \mathrm{~nm}$. The parameters in a solid environment suggest a much stronger temperature dependence of the $R_{\mathrm{A}}$ than observed in this study. This would imply a long-range mechanism for static triplet energy transfer that is not (predominantly) based on exchange interaction and hence a different distance dependence for $k_{\mathrm{A}}$ other than eqn. (19) would apply. A detailed discussion is beyond the scope of this paper.

Basically no reliable values for $A$ and $L$ exist in the literature. From the temperature dependence of the intensity ratio of the excimer and monomer fluorescence of pyrene in ethanol (using data by Stevens and $\mathrm{Ban}^{30}$ ), Butler and Pilling calculated values of $1 \times 10^{14} \mathrm{~s}^{-1} \leq A \leq 2 \times 10^{16} \mathrm{~s}^{-1}$ corresponding to $0.18 \mathrm{~nm} \geq L \geq 0.13 \mathrm{~nm}^{10}$ In comparison, the value of $A$ found in this study is of the right order of magnitude and $L$ is in good agreement.

Based on an encounter-reencounter mechanism ${ }^{31}$ Pilling and Rice showed that the overall bimolecular rate coefficient in the steady-state, $k_{2 \mathrm{~A}}^{\mathrm{BP}}(\infty)=4 \pi R_{\mathrm{A}}^{\mathrm{BP}}(\infty) D$, has two contributions:

$$
k_{2 \mathrm{~A}}^{\mathrm{BP}}(\infty)=k_{2 \mathrm{~A}, T}^{\mathrm{BP}}+k_{2 \mathrm{~A}, D}^{\mathrm{BP}},
$$

with

$$
k_{2 \mathrm{~A}, D}^{\mathrm{BP}}=\frac{4 \pi R D}{I_{0}\left(w_{0}\right)},
$$

which is the rate coefficient for the energy transfer induced by the formation of an encounter pair and

$$
k_{2 \mathrm{~A}, T}^{\mathrm{BP}}=4 \pi R D\left[1-\frac{1}{I_{0}\left(w_{0}\right)}+\frac{L}{R}\left(\gamma+\ln \left(\frac{w_{0}}{2}\right)+\frac{K_{0}\left(w_{0}\right)}{I_{0}\left(w_{0}\right)}\right)\right],
$$

which is the rate coefficient accounting for non-encounter processes. Because of the strong distance dependence of $k_{\mathrm{A}}(r)$, the range of distances at which annihilation takes place is small and centered around $R_{\text {eff }} . I_{0}\left(w_{0}\right)$ and $K_{0}\left(w_{0}\right)$ in eqn. (24) are modified first- and second-type Bessel functions of zero-order and argument $w_{0}$ (eqn. (23)), respectively. Since the exchange interaction depletes excited triplet molecules before the formation of encounter pairs at high viscosities, $k_{2 \mathrm{~A} D}^{\mathrm{BP}}$ is virtually negligible in the present study and $k_{2 \mathrm{~A}}^{\mathrm{BP}}(\infty) \approx \mathrm{k}_{2 \mathrm{~A}, T}^{\mathrm{BP}}$. Fig. 11 shows an Arrhenius plot of $k_{2 \mathrm{~A}}(\infty)$ (circles) as determined from the modified Smoluchowski model, and of $k_{2 \mathrm{AP}}^{\mathrm{BP}}(\infty)$ (triangles) and its contributions $k_{2 \mathrm{~A}, T}^{\mathrm{BP}}$ and $k_{2 \mathrm{~A}, D}^{\mathrm{BP}}$ as calculated from eqn. (24) using an encounter distance of $R \approx 0.7 \mathrm{~nm}$. Fig. 11 essentially demonstrates firstly, that the approximation for $w_{0}>2$ in eqn. (22) holds over the entire temperature range, and secondly, how well $R_{\mathrm{A}}^{\mathrm{BP}}(\infty)$ can be associated with $R_{\mathrm{A}}(\infty)$ from the Smoluchowski model. The activation energy obtained from the Butler and Pilling approach $E_{\mathrm{A}}^{\mathrm{BP}}=39.52 \mathrm{~kJ} \mathrm{~mol}^{-1}$, (frequency factor $A^{\mathrm{BP}}=7.82 \times 10^{20} \mathrm{~s}^{-1}$ ) agrees to within $\approx 8 \%$ with that obtained from the modified Smoluchowski approach $E_{\mathrm{A}}^{\mathrm{Smol}}=36.38 \mathrm{~kJ} \mathrm{~mol}^{-1}\left(A^{\mathrm{Smol}}=0.58 \times 10^{20} \mathrm{~s}^{-1}\right)$. Fig. 11 also includes values of $k_{2 \mathrm{~A}}(\infty)$ obtained from the fits in which $D$ follows exactly a Stokes-Einstein law (open circles, column 4 Table 1). These values do not exhibit Arrhenius behaviour. This again shows that using a fixed $D$ at the corresponding SE value in the fits yield the physically least relevant set of parameters (see Fig. 10).

In essence our measurements corroborate the validity of both, the Butler and Pilling as well as the Smoluchowski model in the steady state regime, if the experimental conditions are chosen appropriately. The essential differences of the two approaches are: (A) The BP model requires two parameters $A$ and $L$ to account for the temperature-dependent description of TTA, the modified Smoluchowski model instead requires only one, i.e. $R_{\mathrm{A}}$. (B) The weak temperature dependence of $R_{\mathrm{A}}$ emerges naturally from the model of Butler and Pilling as opposed to the modified Smoluchowski model which does not include this aspect of $R_{\mathrm{A}}$. (C) The BP model fails to explain the anti-Smoluchowksi time range of TTA, hence no information on $R_{\mathrm{ST}}$ can be obtained from it. (D) The effective steady state annihilation radius as obtained from the Smoluchowski model is of the right order of magnitude, corroborating the activity of a Dexter mechanism, which is the basis of the BP model.

\section{Conclusion}

New measurements of the time-dependent delayed fluorescence of anthracene in $c t$-DMCH are presented in the temperature range between 150 and $134 \mathrm{~K}$. It was shown that an appropriately modified version of Smoluchowski's original theory, as it was introduced in a preceding publication ${ }^{3}$ and applied here to its full extent, describes the kinetics of diffusion-controlled TTA with high accuracy. For the determination of absolute values of the relevant kinetic parameters (annihilation radius $R_{\mathrm{A}}$, Förster radius $R_{\mathrm{ST}}$, triplet decay rate $k_{\mathrm{T}}$ ), the temperature-dependent relative diffusion coefficient $D$ was evaluated based on an appropriate measurement using spatially periodic excitation of the sample. In that way a strong correlation of $D$ and the effective annihilation radius $R_{\mathrm{A}}$ could be diminished. Appropriate fitting procedures for measurements upon spatially homogeneous and spatially periodic excitation were outlined. The validity of the approach was discussed in terms of preconditions for its application (it was found that the binary approximation is valid for experimental conditions presented), and the results obtained regarding the temperature dependence of relevant kinetic parameters. Deviations of $D(T)$ from a Stokes-Einstein law for spherical particles were found. Temperature dependence and size of $R_{\mathrm{A}}, R_{\mathrm{ST}}$ and $k_{\mathrm{T}}$ were physically reasonable and followed expectations. At low viscosity $(\geq 150 \mathrm{~K})$ TTA is likely to be slightly slower than an entirely diffusion-controlled reaction and at high viscosities $(\leq 135 \mathrm{~K})$ effects resulting from the increasing rigidity of the matrix were 
observed. The modified Smoluchowski theory for TTA was finally compared with a model by Butler and Pilling, which is based on a distance-dependent first-order reaction for the annihilation of a triplet pair. The values of the parameters $A$ and $L$ describing the exponential distance dependence of the corresponding rate coefficient were obtained from the temperature dependence of $R_{\mathrm{A}}$ and $D$ in the Smoluchowski approach. The agreement with general values of $A$ and $L$ by Butler and Pilling ${ }^{10}$ was satisfactory.

\section{Acknowledgements}

We would like to thank Dr U. Nickel for comments on the manuscript. P. B. gratefully acknowledges the award of a fellowship by the Alexander von Humboldt-Foundation, Germany.

\section{References}

1 M. v. Smoluchowski, Z. Phys. Chem., 1917, 92, 129-168.

2 S. A. Rice, Diffusion-limited reactions, vol. 25 of the series Comprehensive Chemical Kinetics, ed. C. H. Bamford, C. F. H. Tipper and R. G. Compton, Elsevier, Amsterdam, 1985.

3 B. Nickel, H. E. Wilhelm and C. P. Jaensch, Opt. Spectrosc., 1997, 83, 584-600.

4 B. Nickel, H. E. Wilhelm and A. A. Ruth, Chem. Phys., 1994, 188, 267-287.

5 B. W. Van Der Meer, G. Coker, III and S.-Y. S. Chen, Resonance Energy Transfer - Theory and Data, VCH, Weinheim, 1994.

6 A. A. Ruth, B. Nickel and H. Lesche, Z. Phys. Chem., 1992, 175, 91-108.

7 B. Nickel, Ber. Bunsenges. Phys. Chem., 1972, 76, 582-584.

8 B. Nickel and U. Nickel, Ber. Bunsenges. Phys. Chem., 1972, 76, 584-589.

9 E. G. Meyer and B. Nickel, Z. Naturforsch., Teil A, 1980, 35, 503-520.

10 P. R. Butler and M. J. Pilling, J. Chem. Soc., Faraday Trans. 2, $1977,73,886-894$.
11 H. E. Wilhelm, Anti-Smoluchowski-Zeitverlauf der verzögerten Fluoreszenz aromat-ischer Verbindungen, Dissertation, University of Göttingen, Germany, 1995, p. 32.

12 A. A. Ruth, H. Lesche and B. Nickel, Z. Phys. Chem., 2003, 217, 1-16.

13 D. Klemp and B. Nickel, Chem. Phys., 1993, 174, 297-318.

14 A. A. Ruth, Phosphoreszenz von Phenazin und Chinoxalin in glasbildenden Alkanen: Reorientierung und Spin-Gitter-Relaxation von Molekülen im metastabilen Triplettzustand, Dissertation, University of Göttingen, Germany, 1992.

15 E. R. Pantke and H. Labhart, Chem. Phys. Lett., 1972, 16 , 255-259.

16 G. Greiner, J. Photochem. Photophys. A, 2000, 137, 1-7.

17 W. H. Press, S. A. Teukolsky, W. T. Vetterling and B. P. Flannery, Numerical Recipes in Fortran. The Art of Scientific Computing, Cambridge University Press, 1992, ch. 10, p. 387.

18 J. Jasny, B. Nickel and P. Borowicz, accepted with J. Opt. Soc. Am. B., 2004.

19 A. H. Alwatter, M. D. Lumb and J. B. Birks, in Organic Molecular Photophysics, ed. J. B. Birks, Wiley Interscience, London, 1973, vol. I, p. 403.

20 J. Bartos and J. Kristiak, IOP Publishing. J. Phys.-Condensed Matter, 1999, 11, A371-377.

21 K. Rah and B. C. Eu, Phys Rev. Lett. - 065901, 2002, 8806, $5901 / 1-4$.

22 D. L. Dexter, J. Chem. Phys., 1953, 21, 836-850.

23 A. Kawski, B. Kukliński and P. Bojarski, Z. Naturforsch., Teil A, 2002, 57, 716-722.

24 M. J. Pilling and S. A. Rice, J. Chem. Soc., Faraday Trans. 2, 1976, 72, 792-801

25 B. Nickel, Chem. Phys., 1995, 198, 353-371.

26 M. Inokuti and F. Hirayama, J. Chem. Phys., 1965, 43, 1978-1989.

27 A. N. Terenin and V. L. Ermolaev, Trans. Faraday Soc., 1956, 52, 1042-1052

28 C. Jänsch, Anwendung von Smoluchowskis Theorie auf die Kinetik der diffusionskontrollierten Triplett-Triplett-Annihilation aromatischer Verbindungen, Dissertation, University of Göttingen, Germany, 1997, ISBN 3-89588-961-X.

29 N. A. Efremov, S. G. Kulikov, R. I. Personov and Y. V. Romanovskii, Chem. Phys., 1988, 128, 9-21.

30 B. Stevens and M. I. Ban, Mol. Cryst., 1968, 4, 173-181.

31 R. M. Noyes, J. Am. Chem. Soc., 1956, 78, 5486-5490. 